\title{
Grand unified theories in renormalisable, classically scale invariant gravity
}

\author{
Martin B. Einhorn ${ }^{a, 1}$ and D.R. Timothy Jones ${ }^{a, b}$ \\ ${ }^{a}$ Kavli Institute for Theoretical Physics, University of California, \\ Santa Barbara, CA 93106-4030, U.S.A. \\ ${ }^{b}$ Dept. of Mathematical Sciences, University of Liverpool, \\ Liverpool L69 3BX, U.K. \\ E-mail: meinhorn@umich.edu, drtj@liv.ac.uk
}

Abstract: We analyze $\mathrm{SO}(N)$ and $\mathrm{SU}(N)$ gauge theories with scalars in adjoint and fundamental representations, coupled to renormalisable, classically scale invariant gravity. In the specific case of $\mathrm{SO}(12)$, we show that the quantum field theory can be can be asymptotically free in all couplings (hence ultra-violet complete). For a region of parameter space, Dimensional Transmutation occurs, with the adjoint vacuum expectation value breaking $\mathrm{SO}(12) \rightarrow \mathrm{SU}(6) \otimes \mathrm{U}(1)$ and producing a Low Energy Effective Theory having Einstein-Hilbert gravity. We verify that certain minima are locally stable and lie within the catchment basin of the ultraviolet fixed points.

KEYwords: Models of Quantum Gravity, Renormalization Group, Spontaneous Symmetry Breaking

ArXIV EPRINT: 1908.01400

\footnotetext{
${ }^{1}$ Also at: Michigan Center for Theoretical Physics, Ann Arbor, MI 48109, U.S.A.
} 


\section{Contents}

1 Introduction $\quad 1$

2 The $\mathrm{SO}(N)$ theory 4

3 The $\mathrm{SO}(N) \boldsymbol{\beta}$-functions 4

3.1 Classical gravity 4

3.2 Gravitational corrections 5

4 The SO(12) fixed points $\quad 8$

5 The $\mathrm{SO}(N)$ large $N$ limit $\quad 9$

5.1 Gravitational coupling rescaling-A $\quad 9$

$\begin{array}{lll}5.2 & \text { Gravitational coupling rescaling-B } & 10\end{array}$

6 The $\mathrm{SU}(N)$ theory 11

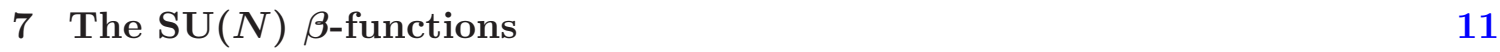

$\begin{array}{lll}7.1 & \text { Classical gravity } & 11\end{array}$

$\begin{array}{lll}7.2 & \text { Gravitational corrections } & 12\end{array}$

$\begin{array}{lll}8 & \mathrm{SU}(9) \text { fixed points } & 12\end{array}$

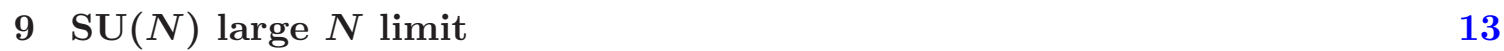

$\begin{array}{lll}9.1 & \text { Gravitational coupling rescaling-A } & 13\end{array}$

$\begin{array}{lll}9.2 & \text { Gravitational coupling rescaling-B } & 14\end{array}$

10 Dimensional transmutation in the $\mathrm{SO}(N)$ model $\quad 15$

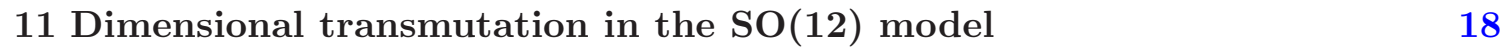

$\begin{array}{lr}12 \text { Summary and conclusions } & 20\end{array}$

A Reduced $\beta$-functions and result for $\varpi_{2} \quad 21$

\section{Introduction}

The search for a successful unification of the Standard Model (SM) with gravitational interactions remains a major preoccupation for theoretical physicists. Most effort for over the last thirty years has been expended on string theory; with results often of considerable mathematical elegance but as yet unproven physical relevance. There are other possibilities, 
however. In some ways the most conceptually simple of these (in that it preserves the renormalisability characteristic of the SM) is renormalisable quantum gravity RQG [1], having a Lagrangian containing no more than two powers of the curvature. A subset of such models is asymptotically free $(\mathrm{AF})$ in the gravitational interactions [2-4]. (Refs. $[5,6]$ provide comprehensive reviews up to their publication dates.) If these are to include matter in a manner in which all their couplings are also AF, the content of such models is rather strictly circumscribed. First of all, it is well known that, in flat space, only non-Abelian gauge theories can be AF in their gauge couplings, and only within a certain subset of models having additional scalars and fermions. Further restrictions are necessary in order to have all the matter couplings AF as well [7]. Similarly, with renormalisable quantum gravity (RQG), there is only a narrowly restricted subset of such non-Abelian gauge theories that remain $\mathrm{AF}$ in all couplings $[8,9]$.

An alternative approach, given the renormalisable nature of RQG, is that of Asymptotic Safety, (AS) [10]-[16]. Originally suggested by Weinberg [10], the key idea of AS for a quantum field theory is the existence of ultra-violet fixed points (UVFP) for the coupling constants, rendering the theory UV complete. As mentioned above, it is well-known that the gravitational couplings are AF for a range of couplings, so it is natural to reconsider these models in the context of AS. In refs. [11, 12], Niedermaier introduced a new scheme for renormalising the Planck mass $M_{P}$ (or Newton's constant $G_{N}$ ) and the cosmological constant $\Lambda$. These, like all coupling constants with positive mass dimension, are UV irrelevant in conventional perturbation theory. Instead, in Niedermaier's nonminimal renormalisation scheme, they acquire anomalous dimensions such that they approach finite nonzero values asymptotically. Even more remarkably, he showed that these UVFPs can be calculated in perturbation theory, and, since the usual dimensionless couplings are AF, these new UVFPs can be determined independently of those couplings. In ref. [12], he argued that his one-loop results were unique and gauge-invariant. He also showed that the same construction can also be applied to nonrenormalisable Einstein-Hilbert (E-H) gravity, but that the results are unavoidably gauge-dependent. This perturbative approach has not to our knowledge been extended to higher loops or to include matter fields, although both may be possible. ${ }^{1}$.

By contrast, in our work, we have followed the usual path of perturbative renormalisation, employing minimal subtraction, for values of the gravitational couplings that are AF, but for which the ratios of dimensionless couplings approach UVFPs. We consider the possibility that $M_{P}$ and $\Lambda$ arise dynamically as the couplings increase at lower energy scales, the result of a form of dimensional transmutation (DT) that is still calculable perturbatively. In fact, this can occur even in pure gravity, but unfortunately we found that the extremum was always locally unstable. As we shall discuss subsequently, in the past and at present, we have focused on whether, through interactions between gravity and matter, AF for all dimensionless couplings and DT may be preserved but some extrema become locally stable for some range of parameters.

Attempts have been made to extend renormalisability to conformal or Weyl gravity,

\footnotetext{
${ }^{1}$ For some recent work on AS, see refs. [13-15], and for references to work up to 2017, see ref. [16]
} 
in which the only quadratic curvature term is the square of the Weyl tensor. In general, such models are only conformal classically, with the symmetry broken in the associated QFT because of the conformal anomaly. One may hope that extensions of such models that have local supersymmetry, for both gravity and matter, may in fact not have such an anomaly. [17-19]. This remains controversial because, in the absence of a regulator that respects all symmetries, it is not clear that, beyond one-loop order, conformal invariance can always be restored on-shell $[18,19] .^{2}$

Our interests are not in a conformal version of these models having zero beta-functions. In the absence of anything but quadratic curvature terms, RQG is classically scale invariant, but the associated QFT does have a conformal anomaly that breaks this global symmetry. This suggests that an interesting subset of models may be those having no mass parameters in the action, i.e., possessing only terms that are classically scale-invariant [20, 21]. Masses will be generated by DT, either nonperturbatively as in Yang-Mills theory or perturbatively in a manner somewhat analogous to massless electrodynamics [22]. We are especially interested in models that have all couplings AF [23-25] in perturbation theory.

In a previous paper [25], we analysed an $\mathrm{SO}(10)$ gauge theory coupled to RQG, including a scalar multiplet in the adjoint representation. We showed, that if we impose scale invariance on the classical theory, it can undergo DT and that there is a region of parameter space such that the couplings are all $\mathrm{AF},{ }^{3}$ remaining perturbatively small at scales down to the point where DT occurs. At this scale the vacuum expectation value (vev) of the scalar multiplet breaks $\mathrm{SO}(10)$ to $\mathrm{SU}(5) \otimes \mathrm{U}(1)$, and the effective theory at lower scales has an Einstein $R$ term arising from the dimensionless nonminimal coupling of scalars to gravity.

Elsewhere [26], we revisited the classic flat space $\beta$-function calculations of CEL [7], which were motivated by the search for asymptotically free (and hence UV complete) nonAbelian gauge theories. We found a number of errors in the CEL results, including the cases of both a $\mathrm{SO}(N)$ and a $\mathrm{SU}(N)$ gauge theory coupled to two scalar multiplets: an adjoint and a fundamental. Here we extend those results to classically scale invariant RQG to see whether the UV completeness and DT phenomena we identified in [25] persist for these cases, which we regard as prototypes for a realistic Grand Unified Theory (GUT) due to the more complicated scalar sector. We consider in detail both the case $\mathrm{SO}(12)$ and the limit of large $N$ for both $\mathrm{SO}(N)$ and $\mathrm{SU}(N)$.

\footnotetext{
${ }^{2}$ In fact, there is evidence to the contrary at two-loop order [19]. Note that with supergravity, most authors do not include quadratic terms but deal only with the effective field theory having the locally supersymmetric counterpart of the Einstein-Hilbert action plus matter. In this respect, the two references $[18,19]$ are exceptional.

${ }^{3}$ To obtain asymptotic freedom of all the matter couplings it is necessary for the one-loop contribution to the gauge $\beta$-function be (nearly) as small in magnitude as possible, as well as, obviously, having the correct sign. This we assumed results from an appropriate contribution to it from fermions.
} 


\section{The $\operatorname{SO}(N)$ theory}

The action we shall consider is

$$
S_{\text {tot }}=\int d^{4} x \sqrt{g}\left[-\frac{1}{4}\left(G_{\mu \nu}^{a}\right)^{2}-\frac{1}{2} g^{\mu \nu} D_{\mu} \phi^{a} D_{\nu} \phi^{a}-\frac{1}{2} g^{\mu \nu} D_{\mu} \chi^{i} D_{\nu} \chi^{i}-V(\phi, \chi)\right]+S_{\text {grav }}
$$

where

$$
S_{\text {grav }}=\int d^{4} x \sqrt{g}\left[\frac{C^{2}}{2 a}+\frac{R^{2}}{3 b}+c G .\right]
$$

Here $C$ is the Weyl tensor and $G$ the Gauss-Bonnet term; $a, b, c$ are dimensionless coupling constants.

All the fields are real. $\chi^{i}$ is in the defining (fundamental) representation with $i: 1 \cdots N$ and $\phi^{a}$ is in the adjoint representation with $a: 1 \cdots N(N-1) / 2$. It is convenient for some purposes to write $\phi^{a}$ as $\Phi=T^{a} \phi^{a}$ where $T^{a}$ are the $N \times N$ hermitian generators of $\operatorname{SO}(N)$, normalised so that

$$
\operatorname{Tr}\left[T^{a} T^{b}\right]=\frac{1}{2} \delta^{a b} .
$$

For an arbitrary irreducible representation of the generators $R^{a}$, we write

$$
\operatorname{Tr}\left[R^{a} R^{b}\right]=T_{R} \delta^{a b} .
$$

$T_{R}$ is determined for each representation by the normalisation eq. (2.3).

The general form of the scale invariant scalar potential is ${ }^{4}$

$$
V(\Phi, \chi)=\frac{\lambda_{1}}{2} T_{2}^{2}+\lambda_{2} T_{4}+\frac{\lambda_{3}}{8}\left(\chi^{t} \chi\right)^{2}+\frac{\lambda_{4}}{2} T_{2}\left(\chi^{t} \chi\right)+\frac{\lambda_{5}}{4} \chi^{t} \Phi^{2} \chi-\xi_{1} T_{2} R-\frac{1}{2} \xi_{2} \chi^{t} \chi R,
$$

where $T_{m}=\operatorname{Tr}\left[\Phi^{m}\right]=\operatorname{Tr}\left[\left(\phi_{a} T^{a}\right)^{m}\right]$. In particular, $T_{2}=\operatorname{Tr}\left[\left(\phi_{a} T^{a}\right)^{2}\right]=\frac{1}{2} \sum_{a}\left(\phi_{a}\right)^{2}$.

\section{$3 \quad$ The $\operatorname{SO}(N) \beta$-functions}

\subsection{Classical gravity}

Although quantum corrections due to gravity are not included in this approximation, the $\xi_{1,2}$ terms in the potential, eq. (2.5), must still be present since, even in a background gravitational field having $R \neq 0, \xi_{1,2}$ undergo renormalisation.

The flat space $\beta$-functions are given in eq. (3.1). (We suppress throughout a factor of $\left(16 \pi^{2}\right)^{-1}$ in each $\beta$-function.)

$$
\begin{aligned}
\beta_{g^{2}}= & -b_{g}\left(g^{2}\right)^{2}, b_{g} \equiv 2\left(\frac{11}{3} C_{G}-\frac{1}{6} T_{S}-\frac{2}{3} T_{F}\right), \\
\beta_{\lambda_{1}}^{(0)}= & \frac{(N(N-1)+16)}{2} \lambda_{1}^{2}+2(2 N-1) \lambda_{1} \lambda_{2}+6 \lambda_{2}^{2}+N \lambda_{4}^{2}+\lambda_{4} \lambda_{5} \\
& +9 g^{4}-6(N-2) g^{2} \lambda_{1}, \\
\beta_{\lambda_{2}}^{(0)}= & (2 N-1) \lambda_{2}^{2}+12 \lambda_{1} \lambda_{2}+\frac{\lambda_{5}^{2}}{8}+\frac{3(N-8) g^{4}}{2}-6(N-2) g^{2} \lambda_{2},
\end{aligned}
$$

\footnotetext{
${ }^{4}$ CEL's adjoint has $\phi \equiv \phi_{a} B^{a}$, with normalization $\operatorname{Tr}\left[\phi^{2}\right]=-\phi_{a} \phi_{a} / 2=-T_{2} / 2 .\left(B_{a}=i R_{a}, \phi=i \Phi / \sqrt{2}\right.$. $)$
} 


$$
\begin{aligned}
\beta_{\lambda_{3}}^{(0)}= & (N+8) \lambda_{3}^{2}+(N-1)\left(\frac{N \lambda_{4}^{2}}{2}+\frac{\lambda_{4} \lambda_{5}}{2}+\frac{\lambda_{5}^{2}}{16}\right)+\frac{3(N-1) g^{4}}{4}-3(N-1) g^{2} \lambda_{3}, \\
\beta_{\lambda_{4}}^{(0)}= & 4 \lambda_{4}^{2}+\lambda_{4}\left[+\frac{(N(N-1)+4)}{2} \lambda_{1}+(2 N-1) \lambda_{2}+(N+2) \lambda_{3}\right] \\
& +\lambda_{5}\left[\frac{(N-1)}{4} \lambda_{1}+\frac{1}{2} \lambda_{2}+\frac{1}{2} \lambda_{3}\right]+\frac{1}{8} \lambda_{5}^{2}+\frac{3 g^{4}}{2}-\frac{3(3 N-5) g^{2} \lambda_{4}}{2}, \\
\beta_{\lambda_{5}}^{(0)}= & \frac{N}{4} \lambda_{5}^{2}+\lambda_{5}\left[2 \lambda_{1}+(N-1) \lambda_{2}+2 \lambda_{3}+8 \lambda_{4}\right]+3(N-4) g^{4}-\frac{3(3 N-5) g^{2} \lambda_{5}}{2} .
\end{aligned}
$$

In eq. (3.1a), $T_{S, F}$ represent the values of $T_{R}$ for the scalars and fermions. For $\mathrm{SO}(N)$, $C_{G}=(N-2) / 2$. For this model, $T_{S}=(N-2) / 2+1 / 2=(N-1) / 2$, so

$$
b_{g}=\frac{21 N-43}{6}-\frac{4}{3} T_{F}
$$

Although $\left\{\xi_{1}, \xi_{2}\right\}$ are not relevant for flat space, in curved spacetime, matter selfinteractions contribute to their one-loop $\beta$ functions. Letting $\xi_{i}^{\prime} \equiv \xi_{i}+1 / 6$, we have

$$
\begin{aligned}
& \beta_{\xi_{1}^{\prime}}^{(0)}=\beta_{11} \xi_{1}^{\prime}+\beta_{12} \xi_{2}^{\prime}, \\
& \beta_{\xi_{2}^{\prime}}^{(0)}=\beta_{21} \xi_{1}^{\prime}+\beta_{22} \xi_{2}^{\prime},
\end{aligned}
$$

where

$$
\begin{aligned}
& \beta_{11}=\left[\frac{N(N-1)+4}{2} \lambda_{1}+(2 N-1) \lambda_{2}-3(N-2) \alpha\right], \\
& \beta_{12}=\left[N \lambda_{4}+\frac{1}{2} \lambda_{5}\right], \\
& \beta_{21}=\frac{(N-1)}{2}\left[N \lambda_{4}+\frac{1}{2} \lambda_{5}\right], \\
& \beta_{22}=\left[(N+2) \lambda_{3}-\frac{3(N-1)}{2} \alpha\right] .
\end{aligned}
$$

Here (and subsequently) we have set $\alpha \equiv g^{2}$. eq. (3.3) obviously gives rise to fixed point solutions for $\beta_{\xi_{1,2}^{\prime}}^{(0)}$ corresponding to conformal coupling, $\xi_{1}^{\prime}=\xi_{2}^{\prime}=0$. Assuming that $\operatorname{det}\left[\beta_{i j}\right] \neq$ 0 , there are no other fixed point solutions. We shall see shortly that the one-loop gravity contribution changes that result.

\subsection{Gravitational corrections}

The gravitational corrections to the matter $\beta$-functions have a universal form. We infer ${ }^{5}$ from ref. [20] that the full $\beta$-functions are

$$
\begin{aligned}
& \beta_{\lambda_{1}}=\beta_{\lambda_{1}}^{(0)}+\Delta \beta^{(1)}\left(\xi_{1}^{\prime}\right)+\lambda_{1} \Delta \beta^{(2)}\left(\xi_{1}^{\prime}\right), \\
& \beta_{\lambda_{2}}=\beta_{\lambda_{2}}^{(0)}+\lambda_{2} \Delta \beta^{(2)}\left(\xi_{1}^{\prime}\right),
\end{aligned}
$$

\footnotetext{
${ }^{5} \mathrm{~A}$ word of caution concerning ref. [20]: at the time of our writing, the journal version differs from the earlier arXiv version, correcting some formulas.
} 


$$
\begin{aligned}
& \beta_{\lambda_{3}}=\beta_{\lambda_{3}}^{(0)}+\Delta \beta^{(1)}\left(\xi_{2}^{\prime}\right)+\lambda_{3} \Delta \beta^{(2)}\left(\xi_{2}^{\prime}\right), \\
& \beta_{\lambda_{4}}=\beta_{\lambda_{4}}^{(0)}+\Delta \beta^{(4)}\left(\xi_{1}^{\prime}, \xi_{2}^{\prime}\right)+\lambda_{4} \Delta \beta^{(3)}\left(\xi_{1}^{\prime}, \xi_{2}^{\prime}\right), \\
& \beta_{\lambda_{5}}=\beta_{\lambda_{5}}^{(0)}+\lambda_{5} \Delta \beta^{(3)}\left(\xi_{1}^{\prime}, \xi_{2}^{\prime}\right), \\
& \beta_{\xi_{j}^{\prime}}=\beta_{\xi_{j}^{\prime}}^{(0)}+\Delta \beta_{\xi_{j}^{\prime}}\left(\xi_{j}^{\prime}\right),
\end{aligned}
$$

where

$$
\begin{aligned}
\Delta \beta^{(1)}\left(\xi^{\prime}\right) & =a^{2}\left(\xi^{\prime}-\frac{1}{6}\right)^{2}\left[5+9 \xi^{2} x^{2}\right], \\
\Delta \beta^{(2)}\left(\xi^{\prime}\right) & =a\left(5-18 x \xi^{\prime 2}\right), \\
\Delta \beta^{(3)}\left(\xi_{1}^{\prime}, \xi_{2}^{\prime}\right) & =a\left[5-3 x\left(\xi_{1}^{\prime 2}+\xi_{2}^{\prime 2}+4 \xi_{1}^{\prime} \xi_{2}^{\prime}\right)\right], \\
\Delta \beta^{(4)}\left(\xi_{1}^{\prime}, \xi_{2}^{\prime}\right) & =a^{2}\left(\xi_{1}^{\prime}-\frac{1}{6}\right)\left(\xi_{2}^{\prime}-\frac{1}{6}\right)\left[5+9 x^{2} \xi_{1}^{\prime} \xi_{2}^{\prime}\right], \\
\Delta \beta_{\xi^{\prime}}\left(\xi^{\prime}\right) & =a\left(\xi^{\prime}-\frac{1}{6}\right)\left(\frac{10}{3 x}-\frac{3}{2} \xi^{\prime}\left(2 \xi^{\prime}+1\right) x\right) .
\end{aligned}
$$

The $\beta$-function for the gauge coupling is unchanged by gravity (at one loop) and remains as in eq. (3.1a).

For the gravitational self-interactions we have (again of universal form):

$$
\begin{array}{lll}
\beta_{a}=-b_{2} a^{2}, & \beta_{b}=-a^{2} b_{3}\left(x, \xi_{1}^{\prime}, \xi_{2}^{\prime}\right), & \beta_{c}=-b_{1}, \\
b_{2}=\frac{133}{10}+N_{a}, & b_{3} \equiv \frac{10}{3}-5 x+\frac{5 x^{2}}{12}+\left(\frac{N(N-1)}{2} \xi_{1}^{\prime 2}+N \xi_{2}^{\prime 2}\right) \frac{3 x^{2}}{2}, & b_{1}=\frac{196}{45}+N_{c},
\end{array}
$$

where $N_{a}=\left[N_{0}+6 N_{1 / 2}+12 N_{1}^{0}+13 N_{1}\right] / 60 ; N_{c}=\left[N_{0}+11 N_{1 / 2}+62 N_{1}^{0}+63 N_{1}\right] / 360$. Here $N_{0}$ denotes the number of (real) scalars; $N_{1 / 2}$, Dirac fermions; $N_{1}^{0}$, massless vectors; $N_{1}$, massive vectors. (For chiral or Majorana fermions, the coefficients of $N_{1 / 2}$ would be half those given above). In the last equation, we set $x \equiv b / a=1 / w$. It is sometimes convenient to use $x$ instead of $b$, with

$$
\beta_{x}=a\left(b_{2} x-b_{3}\right) .
$$

We shall eventually be interested in determining whether this model is asymptotically free in all couplings, requiring reduced couplings to approach UVFP's. In this case, we have $N_{0}=N(N-1) / 2+N, N_{1}^{0}=N(N-1) / 2$, so $N_{a} \geq(13 N(N-1) / 2+N) / 60$. For $N \geq 10$, $N_{a} \geq 9.92$. So $b_{2} \gtrsim 23.22$.

To analyse the $\beta$-functions and seek FPs, it is convenient to introduce rescaled couplings $\bar{a} \equiv a / \alpha, x_{i} \equiv \lambda_{i} / \alpha$, with consequent "reduced" $\beta$-functions,

$$
\bar{\beta}_{\bar{a}} \equiv \frac{d \bar{a}}{d u}=\bar{a}\left(b_{g}-b_{2} \bar{a}\right), \bar{\beta}_{x} \equiv \frac{d x}{d u}=\bar{a}\left(b_{2} x-b_{3}\right), \bar{\beta}_{x_{i}} \equiv \frac{d x_{i}}{d u}, \quad \text { where } d u \equiv \alpha(t) d t .
$$

For $b_{g}>0$, as is required for asymptotic freedom of $\alpha$, we see that $\bar{a}$ has a UVFP at

$$
\bar{a}_{F P}=b_{g} / b_{2}
$$


Hence $a \rightarrow 0$ at high energies, and, since UVFPs only exist when $b_{g}$ is small, $\bar{a}_{F P}$ is small. The $\bar{\beta}_{x_{i}}$ are given by

$$
\begin{aligned}
\bar{\beta}_{x_{1}}= & \left(\frac{N(N-1)+16}{2}\right) x_{1}^{2}+6 x_{2}^{2}+2(2 N-1) x_{1} x_{2}+N x_{4}^{2}+x_{4} x_{5} \\
& +\left(b_{g}-6(N-2)\right) x_{1}+9+\overline{\Delta \beta}^{(1)}\left(\xi_{1}^{\prime}\right)+x_{1} \overline{\Delta \beta}^{(2)}\left(\xi_{1}^{\prime}\right) \\
\bar{\beta}_{x_{2}}= & (2 N-1) x_{2}^{2}+12 x_{1} x_{2}+\frac{1}{8} x_{5}^{2}+\left(b_{g}-6(N-2)\right) x_{2} \\
& +\frac{3(N-8)}{2}+x_{2} \overline{\Delta \beta}^{(2)}\left(\xi_{1}^{\prime}\right) \\
\bar{\beta}_{x_{3}}= & (N+8) x_{3}^{2}+\frac{N(N-1)}{2} x_{4}^{2}+\frac{N-1}{16} x_{5}^{2}+\frac{N-1}{2} x_{4} x_{5} \\
& +\left(b_{g}-3(N-1)\right) x_{3}+\frac{3(N-1)}{4}+\overline{\Delta \beta}^{(1)}\left(\xi_{2}^{\prime}\right)+x_{3} \overline{\Delta \beta}^{(2)}\left(\xi_{2}^{\prime}\right) \\
\bar{\beta}_{x_{4}}= & 4 x_{4}^{2}+\frac{1}{8} x_{5}^{2}+x_{5}\left[\frac{N-1}{4} x_{1}+\frac{1}{2} x_{2}+\frac{1}{2} x_{3}\right]+x_{4}\left[\left(\frac{N(N-1)}{2}+2\right) x_{1}+(2 N-1) x_{2}\right. \\
& \left.+(N+2) x_{3}+b_{g}-\frac{3(3 N-5)}{2}\right]+\frac{3}{2}+\bar{\Delta}^{(4)}\left(\xi_{1}^{\prime}, \xi_{2}^{\prime}\right)+x_{4} \overline{\Delta \beta}^{(3)}\left(\xi_{1}^{\prime}, \xi_{2}^{\prime}\right) \\
\bar{\beta}_{x_{5}}= & \frac{N}{4} x_{5}^{2}+x_{5}\left[2 x_{1}+(N-1) x_{2}+2 x_{3}+8 x_{4}+b_{g}-\frac{3(3 N-5)}{2}\right] \\
& +3(N-4)+x_{5} \overline{\Delta \beta}^{(3)}\left(\xi_{1}^{\prime}, \xi_{2}^{\prime}\right) .
\end{aligned}
$$

In eq. (3.11), the $\overline{\Delta \beta}$ terms are identical to the $\Delta \beta$ terms in eq. (3.6), except that $a$ is replaced by $\bar{a}$.

In terms of reduced couplings, the classical gravity $\beta$-functions for $\beta_{\xi_{1,2}^{\prime}}$ become

$$
\begin{aligned}
& \bar{\beta}_{\xi_{1}^{\prime}}^{(0)}=\bar{\beta}_{11} \xi_{1}^{\prime}+\bar{\beta}_{12} \xi_{2}^{\prime}, \\
& \bar{\beta}_{\xi_{2}^{\prime}}^{(0)}=\bar{\beta}_{21} \xi_{1}^{\prime}+\bar{\beta}_{22} \xi_{2}^{\prime},
\end{aligned}
$$

where

$$
\begin{aligned}
& \bar{\beta}_{11}=\left[\frac{N(N-1)+4}{2} x_{1}+(2 N-1) x_{2}-3(N-2)\right], \\
& \bar{\beta}_{12}=\left[N x_{4}+\frac{1}{2} x_{5}\right], \\
& \bar{\beta}_{21}=\frac{(N-1)}{2}\left[N x_{4}+\frac{1}{2} x_{5}\right], \\
& \bar{\beta}_{22}=\left[(N+2) x_{3}-\frac{3(N-1)}{2}\right] .
\end{aligned}
$$

Adding gravitational corrections, we have

$$
\bar{\beta}_{\xi_{j}^{\prime}}=\bar{\beta}_{\xi_{j}^{\prime}}^{(0)}+\overline{\Delta \beta}_{\xi_{j}^{\prime}}\left(\xi_{j}^{\prime}\right),
$$


where $\overline{\Delta \beta}_{\xi^{\prime}}\left(\xi^{\prime}\right)$ is given by eq. (3.6e) with $a \rightarrow \bar{a}$ :

$$
\overline{\Delta \beta}_{\xi^{\prime}}\left(\xi^{\prime}\right)=\bar{a}\left(\xi^{\prime}-\frac{1}{6}\right)\left(\frac{10}{3 x}-\frac{3}{2} \xi^{\prime}\left(2 \xi^{\prime}+1\right) x\right) .
$$

While the matter contributions $\bar{\beta}_{\xi_{j}^{\prime}}^{(0)}$ vanish for conformal coupling $\left(\xi^{\prime}=0\right.$ or $\left.\xi=-1 / 6\right)$, the gravitational corrections vanish for minimal coupling, $\left(\xi^{\prime}=+1 / 6\right.$ or $\left.\xi=0\right)$. When the two are combined, the FP respects neither limit.

\section{The $\mathrm{SO}(12)$ fixed points}

Our first goal in this class of theories is the identification of cases such that the renormalisation group evolution of the couplings approaches a fixed point at high energies (UVFP). In the flat space limit, we showed that a minimum value of $\mathrm{N}=12$ is required. For $N=12$ and with the minimum possible value of $b_{g}$, which is $b_{g}=1 / 6$, we found a UVFP with results for the quartic couplings in the flat space limit as follows:

$$
\begin{array}{ll}
x_{1}=0.262953, & x_{2}=0.111668, \\
x_{4}=0.104270, & x_{5}=0.581883 .
\end{array} \quad x_{3}=0.376914,
$$

As mentioned earlier, even with classical gravity, $\xi_{1,2}^{\prime}$ both nevertheless undergo renormalisation. However, it is easy to see from eq. (3.3) that they have a FP for the conformal values

$$
\xi_{1}^{\prime}=\xi_{2}^{\prime}=0
$$

For curved space we expect to find a FP with similar values of the quartic couplings and values of $\xi_{1,2}^{\prime}$ both close to the conformal value (zero). This is basically because, as explained in ref. [25], the FP result for $a / \alpha$ is necessarily small. The result for $N=12$ is

$$
\begin{aligned}
x_{1} & =0.263283, & & x_{2}=0.111708, \\
x_{4} & =.104565, & & x_{3}=0.377518, \\
\xi_{1}^{\prime} & =-1.41379 \times 10^{-6}, & \xi_{2}^{\prime}=-2.00257 \times 10^{-6} & \\
x & =153.548 & & \\
\bar{a} & =1 / 354 . & &
\end{aligned}
$$

The precise values of $\bar{a}, x$ depend on the nature of the fermion content. To achieve $b_{g}=1 / 6$, we have taken 52 two-component fermions in the fundamental representation, so that $N_{\frac{1}{2}}=$ $26 N=312 .{ }^{6}$ Then with $N_{1}^{0}=66$ and $N_{0}=78$, we have $b_{2}=59$, and $\bar{a}=b_{g} / b_{2}=1 / 354$.

Note that the large value of $x$ at the FP does not invalidate perturbation theory since $b=a x$ is only $O(1)$ at the FP.

There is another FP with similar values of $x_{i}, \xi_{1,2}^{\prime}$ and a small value of $x$, which is a saddle point. We shall not elaborate on that.

\footnotetext{
${ }^{6}$ Even for fixed $b_{g}$, there are several possible choices for the fermion representations, so the precise value of $N_{\frac{1}{2}}$ can vary somewhat. It is clear, however, from eqs. (3.7a), (3.10) that any such choice results in a relatively large value for $b_{2}$, and consequently a small value for $\bar{a}$. Thus, the results eq. (4.3) will be rather insensitive to such variations in $N_{\frac{1}{2}}$.
} 


\section{The $\operatorname{SO}(N)$ large $N$ limit}

As we have seen, requiring the existence of a UVFP leads to a minimum value of $N$. It is therefore natural to consider the large $N$ limit, first discussed in non-abelian gauge theories by 't Hooft. To retain finite couplings in the large $N$ limit, we need to rescale the couplings. Since we cannot solve the theory exactly in this limit, we shall have to require that these rescaled couplings remain perturbatively small at all relevant scales.

Assuming $b_{g}=\widetilde{b}_{g} N$, we found that for the quartic scalar couplings, scaling behavior requires

$$
\alpha=\frac{\widetilde{\alpha}}{N}, \lambda_{1}=\frac{\widetilde{\lambda}_{1}}{N^{2}}, \lambda_{2}=\frac{\widetilde{\lambda}_{2}}{N}, \lambda_{3}=\frac{\widetilde{\lambda}_{3}}{N}, \lambda_{4}=\frac{\widetilde{\lambda}_{4}}{N^{p_{4}}}, \lambda_{5}=\frac{\widetilde{\lambda}_{5}}{N},
$$

for $3 / 2 \leq p_{4} \leq 2$. In ref. [27], we argued that the ambiguity in the rescaling of $\lambda_{4}$ reflects a nonuniformity of the limiting behavior that is best resolved by setting $p_{4}=2$.

\subsection{Gravitational coupling rescaling-A}

For the remaining couplings associated with the gravitational interactions, the natural rescaling takes the form

$$
a=\frac{\widetilde{a}}{N} \alpha, b=\frac{\widetilde{b}}{N} \alpha, c=N \widetilde{c}, \xi_{1}^{\prime}=\widetilde{\xi_{1}^{\prime}}, \xi_{2}^{\prime}=\widetilde{\xi_{2}^{\prime}},
$$

We have incorporated both the rescaling with powers of $N$ and forming ratios of couplings to $\widetilde{\alpha}$ in the above equations. Note that since the scalings of $a$ and $b$ are identical, we have $\widetilde{x}=x$.

Defining $\widetilde{y}_{n} \equiv \widetilde{\lambda}_{n} / \alpha$, the reduced $\beta$-functions in the large $N$ limit are as follows: for the quartic couplings,

$$
\begin{aligned}
& \bar{\beta}_{\widetilde{y}_{1}}=\frac{1}{2} \widetilde{y}_{1}^{2}+6 \widetilde{y}_{2}^{2}+9+\left(4 \widetilde{y}_{2}+\widetilde{b}_{g}-6\right) \widetilde{y}_{1}, \\
& \bar{\beta}_{\widetilde{y}_{2}}=2 \widetilde{y}_{2}^{2}+\frac{3}{2}+\left(\widetilde{b}_{g}-6\right) \widetilde{y}_{2}, \\
& \bar{\beta}_{\widetilde{y}_{3}}=\widetilde{y}_{3}^{2}+\frac{1}{16} \widetilde{y}_{5}^{2}+\frac{3}{4}+\left(\widetilde{b}_{g}-3\right) \widetilde{y}_{3}, \\
& \bar{\beta}_{\widetilde{y}_{4}}=\widetilde{y}_{5}\left[\frac{1}{4} \widetilde{y}_{1}+\frac{1}{2} \widetilde{y}_{2}+\frac{1}{2} \widetilde{y}_{3}\right]+\frac{1}{8} \widetilde{y}_{5}^{2}+\frac{3}{2}+\left[\frac{1}{2} \widetilde{y}_{1}+2 \widetilde{y}_{2}+\widetilde{y}_{3}+\widetilde{b}_{g}-\frac{9}{2}\right] \widetilde{y}_{4}, \\
& \bar{\beta}_{\widetilde{y}_{5}}=\frac{1}{4} \widetilde{y}_{5}^{2}+3+\left(\widetilde{y}_{2}+\widetilde{b}_{g}-\frac{9}{2}\right) \widetilde{y}_{5},
\end{aligned}
$$

and for the gravitational couplings,

$$
\begin{aligned}
\bar{\beta}_{\widetilde{a}} & =\widetilde{a}\left(\widetilde{b}_{g}-\widetilde{b}_{2} \widetilde{a}\right), \\
\bar{\beta}_{\widetilde{x}} & =-\widetilde{a} \widetilde{x}\left(\frac{3}{4} \widetilde{\xi}_{1}^{2} \widetilde{x}-\widetilde{b}_{2}\right), \\
\bar{\beta}_{\widetilde{\xi}_{1}^{\prime}} & =\left(\frac{1}{2} \widetilde{y}_{1}+2 \widetilde{y}_{2}-3\right) \widetilde{\xi}_{1}^{\prime}, \\
\bar{\beta}_{\widetilde{\xi}_{2}^{\prime}} & =\left(\widetilde{y}_{3}-\frac{3}{2}\right) \widetilde{\xi}_{2}^{\prime}+\left(\frac{1}{2} \widetilde{y}_{4}+\frac{1}{4} \widetilde{y}_{5}\right) \widetilde{\xi}_{1}^{\prime} .
\end{aligned}
$$

Here we have defined $\widetilde{b}_{g}=b_{g} / N$ and $\widetilde{b}_{2}=b_{2} / N$. 


\begin{tabular}{|c|c|c|c|c|c|}
\hline$\widetilde{b}_{g}$ & $\widetilde{y}_{1}$ & $\widetilde{y}_{2}$ & $\widetilde{y}_{3}$ & $\widetilde{y}_{4}$ & $\widetilde{y}_{5}$ \\
\hline 0. & 2.64270 & 0.27527 & 0.28941 & 0.97035 & 0.74275 \\
\hline $1 / 6$ & 2.94605 & 0.28499 & 0.31255 & 1.20422 & 0.77847 \\
\hline $1 / 3$ & 3.45350 & 0.29553 & 0.34039 & 1.69063 & 0.81820 \\
\hline $5 / 12$ & 4.08657 & 0.30114 & 0.35661 & 2.51800 & 0.83981 \\
\hline
\end{tabular}

Table 1. Flat space UVFPs for $\mathrm{SO}(\infty)$.

\begin{tabular}{|c|c|c|c|c|c|c|c|}
\hline$\widetilde{b}_{g}$ & $\widetilde{y}_{1}$ & $\widetilde{y}_{2}$ & $\widetilde{y}_{3}$ & $\widetilde{y}_{4}$ & $\widetilde{y}_{5}$ & $x$ & $\widetilde{\xi}_{2}^{\prime}$ \\
\hline 0.40961 & 4.79737 & 0.30066 & 0.35517 & 3.90016 & 0.837923 & $\widetilde{b}_{2} /\left(\widetilde{\xi}_{1}^{\prime}\right)^{2}$ & $1.88636 \widetilde{\xi}_{1}^{\prime}$ \\
\hline 0.40961 & 4.79737 & 0.30066 & 2.23523 & -3.19276 & 0.837923 & $\widetilde{b}_{2} /\left(\widetilde{\xi}_{1}^{\prime}\right)^{2}$ & $1.88636 \widetilde{\xi}_{1}^{\prime}$ \\
\hline
\end{tabular}

Table 2. Curved Space UVFPs for $\mathrm{SO}(\infty)$.

After the rescaling chosen in eqs. (5.1), (5.2), the $\beta$-functions for the quartic couplings are the same as in flat space; hence their values at any FP will be the same as in flat space.

We found UVFPs for a range of values of $\widetilde{b}_{g}$ from eq. (5.3) above in ref. [26] with results as shown in table 1. (There was an calculational error in ref. [26] so that the results in table 1 differ slightly from the corresponding table there). It is easy to see that these remain FPs if the remaining couplings are $\widetilde{\xi_{1}^{\prime}}=\widetilde{\xi_{2}^{\prime}}=\widetilde{x}=0$. However, from eq. (5.4b) we then see that these FPs are destabilised by the gravitational corrections since $\widetilde{b}_{2}>13 / 120$.

Note, however, that the results in table 1 correspond to various specific values of $\widetilde{b}_{g}$. There are a finite possible number of values of $b_{g}$ for any given $N$, but the number of values of $\widetilde{b}_{g}$ tends to infinity in the large $N$ limit. We may therefore allow arbitrary values of $\widetilde{b}_{g}$ in the search for a FP.

We now seek a FP with nonzero $\widetilde{\xi}_{1}^{\prime}$. We may first solve eq. (5.3a), eq. (5.3b) and eq. (5.4c) for $\widetilde{y}_{1}, \widetilde{y}_{2}$ and $\widetilde{b}_{g}$. We can then solve eqs. (5.3c)-(5.3e) for $\widetilde{y}_{3}, \widetilde{y}_{4}, \widetilde{y}_{5}$. This leads to two new FPs, shown in table 2. Unfortunately, neither of these FPs is UV stable.

We also have the possibility $\widetilde{\xi_{1}^{\prime}}=x=0, \widetilde{\xi}_{2}^{\prime} \neq 0$, if $\widetilde{y}_{3}=3 / 2$. In this case however, we find no real FPs.

So it seems that with the most natural rescaling (in terms of powers of $N$ ), gravitational corrections destabilise the UVFP. In the next subsection we explore an alternative rescaling, albeit with a similar conclusion.

\subsection{Gravitational coupling rescaling-B}

There is an alternative rescaling giving rise to a non-trivial large $N$ limit as follows:

$$
a=\frac{\widetilde{a}}{N} \alpha, b=\frac{\widetilde{b}}{N^{3}} \alpha, c=N \widetilde{c}, \xi_{1}^{\prime}=N \widetilde{\xi_{1}^{\prime}}, \xi_{2}^{\prime}=N \widetilde{\xi_{2}^{\prime}},
$$

The quartic coupling rescaling is done in the same way as in the previous subsection. Note that now $\widetilde{x}=N^{2} x$. The resulting reduced $\beta$-functions (in the large $N$ limit) take the form 
(where we have $\widetilde{y}_{n} \equiv \lambda_{n} / \alpha$ as before),

$$
\begin{aligned}
& \bar{\beta}_{\widetilde{y}_{1}=} \frac{1}{2} \widetilde{y}_{1}^{2}+6 \widetilde{y}_{2}^{2}+9+\left(4 \widetilde{y}_{2}+\widetilde{b}_{g}-6\right) \widetilde{y}_{1}+5 \widetilde{a}^{2} \widetilde{\xi}_{1}^{2}, \\
& \bar{\beta}_{\widetilde{y}_{2}}= 2 \widetilde{y}_{2}^{2}+\frac{3}{2}+\left(\widetilde{b}_{g}-6\right) \widetilde{y}_{2}, \\
& \bar{\beta}_{\widetilde{y}_{3}=}=\widetilde{y}_{3}^{2}+\frac{1}{16} \widetilde{y}_{5}^{2}+\frac{3}{4}+\left(\widetilde{b}_{g}-3\right) \widetilde{y}_{3}, \\
& \bar{\beta}_{\widetilde{y}_{4}=}=\widetilde{y}_{5}\left[\frac{1}{4} \widetilde{y}_{1}+\frac{1}{2} \widetilde{y}_{2}+\frac{1}{2} \widetilde{y}_{3}\right]+\frac{1}{8} \widetilde{y}_{5}^{2}+\frac{3}{2} \\
&+\left[\frac{1}{2} \widetilde{y}_{1}+2 \widetilde{y}_{2}+\widetilde{y}_{3}+\widetilde{b}_{g}-\frac{9}{2}\right] \widetilde{y}_{4},+5 \widetilde{a}^{2} \widetilde{\xi}_{1}^{\prime} \widetilde{\xi}_{2}^{\prime}, \\
& \bar{\beta}_{\widetilde{y}_{5}=} \frac{1}{4} \widetilde{y}_{5}^{2}+3+\left(\widetilde{y}_{2}+\widetilde{b}_{g}-\frac{9}{2}\right) \widetilde{y}_{5}, \\
& \bar{\beta}_{\widetilde{\xi}_{1}^{\prime}=} \frac{1}{36}\left(-108+18 \widetilde{y}_{1}+72 \widetilde{y}_{2}\right) \widetilde{\xi}_{1}^{\prime}+\frac{10}{3} \frac{\widetilde{a} \widetilde{\xi}_{1}^{\prime}}{\widetilde{x}}, \\
& \bar{\beta}_{\widetilde{\xi}_{2}^{\prime}=} \frac{1}{36}\left(\left(-54+36 \widetilde{y}_{3}\right) \widetilde{\xi}_{2}^{\prime}+18 \widetilde{\xi}_{1}^{\prime}\left(\widetilde{y}_{4}+\frac{1}{2} \widetilde{y}_{5}\right)\right)+\frac{10}{3} \frac{\widetilde{a} \widetilde{\xi}_{2}^{\prime}}{\widetilde{x}}, \\
& \bar{\beta}_{\widetilde{x}}=-(1 / 12) \widetilde{a}\left(9 \widetilde{x}^{2} \widetilde{\xi}_{1}^{2}-12 \widetilde{b}_{2} \widetilde{x}+40\right) .
\end{aligned}
$$

Unlike the previous case, there is no FP corresponding to table 1 with $\xi_{1}^{\prime}=\xi_{2}^{\prime}=x=0$. However, with $\xi_{1}^{\prime}=\xi_{2}^{\prime}=0$, we do reproduce table 1 with a FP at $\widetilde{x}=10 /\left(3 \widetilde{b}_{2}\right)$. However, once again this is not a UVFP.

\section{The $\mathrm{SU}(N)$ theory}

In this case we have the scalar potential

$$
\begin{aligned}
V(\Phi, \chi)= & \frac{1}{2} \lambda_{1}\left(\operatorname{Tr} \Phi^{2}\right)^{2}+\lambda_{2} \operatorname{Tr} \Phi^{4}+\frac{1}{2} \lambda_{3}\left(\chi_{i}^{\dagger} \chi^{i}\right)^{2}+\lambda_{4} \chi_{i}^{\dagger} \chi^{i} \operatorname{Tr} \Phi^{2}+\lambda_{5} \chi_{i}^{\dagger} \Phi_{k}^{i} \Phi^{k}{ }_{j} \chi^{j} \\
& -\frac{1}{2} \xi_{1} \phi^{2} R-\xi_{2} \chi_{i}^{\dagger} \chi^{i} R .
\end{aligned}
$$

Again $\Phi=T^{a} \phi^{a}$, where now $a=1,2 \ldots N^{2}-1 . \chi^{i}[i=1,2 \ldots N]$ is now a complex multiplet in the defining (fundamental) representation, and $T^{a}$ are no longer (all) antisymmetric; they are again normalised so that

$$
\operatorname{Tr} T^{a} T^{b}=\frac{1}{2}
$$

\section{$7 \quad$ The $\operatorname{SU}(N) \beta$-functions}

\subsection{Classical gravity}

As was the case with $\mathrm{SO}(N)$, the potential eq. (6.1) still must include the $\xi_{1,2}$-terms. The flat space $\beta$-functions are [26]

$$
\begin{aligned}
& \beta_{g^{2}}=-b_{g}\left(g^{2}\right)^{2}, b_{g} \equiv \frac{21 N-1}{3}-\frac{4}{3} T_{F} \\
& \beta_{\lambda_{1}}=\left(N^{2}+7\right) \lambda_{1}^{2}+\frac{4\left(2 N^{2}-3\right)}{N} \lambda_{1} \lambda_{2}+\frac{12\left(N^{2}+3\right)}{N^{2}} \lambda_{2}^{2}+2 N \lambda_{4}^{2}+4 \lambda_{4} \lambda_{5}-12 N g^{2} \lambda_{1}+18 g^{4}
\end{aligned}
$$




$$
\begin{aligned}
\beta_{\lambda_{2}}= & \frac{4\left(N^{2}-9\right)}{N} \lambda_{2}^{2}+12 \lambda_{1} \lambda_{2}+\lambda_{5}^{2}-12 N g^{2} \lambda_{2}+3 N g^{4}, \\
\beta_{\lambda_{3}}= & 2(N+4) \lambda_{3}^{2}+\left(N^{2}-1\right) \lambda_{4}^{2}+\frac{(N-1)\left(N^{2}+2 N-2\right)}{2 N^{2}} \lambda_{5}^{2}+\frac{2\left(N^{2}-1\right)}{N} \lambda_{4} \lambda_{5} \\
& -\frac{6\left(N^{2}-1\right)}{N} g^{2} \lambda_{3}+\frac{3(N-1)\left(N^{2}+2 N-2\right)}{2 N^{2}} g^{4}, \\
\beta_{\lambda_{4}}= & 4 \lambda_{4}^{2}+\lambda_{4}\left[\left(N^{2}+1\right) \lambda_{1}+\frac{2\left(2 N^{2}-3\right)}{N} \lambda_{2}+2(N+1) \lambda_{3}\right] \\
& +\lambda_{5}^{2}+\lambda_{5}\left[\frac{N^{2}-1}{N} \lambda_{1}+\frac{2\left(N^{2}+3\right)}{N^{2}} \lambda_{2}+2 \lambda_{3}\right]-\frac{3\left(3 N^{2}-1\right)}{N} g^{2} \lambda_{4}+3 g^{4}, \\
\beta_{\lambda_{5}}= & \frac{N^{2}-4}{N} \lambda_{5}^{2}+\lambda_{5}\left[2 \lambda_{1}+\frac{2\left(N^{2}-6\right)}{N} \lambda_{2}+2 \lambda_{3}+8 \lambda_{4}-\frac{3\left(3 N^{2}-1\right)}{N} g^{2}\right]+3 N g^{4} .
\end{aligned}
$$

We also have

$$
\begin{aligned}
& \beta_{\xi_{1}^{\prime}}^{(0)}=\beta_{11} \xi_{1}^{\prime}+\beta_{12} \xi_{2}^{\prime}, \\
& \beta_{\xi_{2}^{\prime}}^{(0)}=\beta_{21} \xi_{1}^{\prime}+\beta_{22} \xi_{2}^{\prime},
\end{aligned}
$$

where

$$
\begin{aligned}
& \beta_{11}=\left[\left(N^{2}+1\right) \lambda_{1}+2 \frac{2 N^{2}-3}{N} \lambda_{2}-6 \alpha N\right], \\
& \beta_{12}=\left[2 N \lambda_{4}+\lambda_{5}\right], \\
& \beta_{21}=\left[\left(N^{2}-1\right) \lambda_{4}+\frac{N^{2}-1}{2 N} \lambda_{5}\right], \\
& \beta_{22}=\left[(2 N+2) \lambda_{3}-3 \frac{N^{2}-1}{N} \alpha\right] .
\end{aligned}
$$

\subsection{Gravitational corrections}

As we indicated in the $\mathrm{SO}(N)$ discussion above, the gravitational corrections to the matter $\beta$-functions have a universal form; eq. (3.5) remains valid in the $\mathrm{SU}(N)$ case. There are minor changes to the gravitational corrections so that we have:

$$
\begin{array}{lll}
\beta_{a}=-b_{2} a^{2}, & \beta_{b}=-a^{2} b_{3}\left(x, \xi_{1}^{\prime}, \xi_{2}^{\prime}\right), & \beta_{c}=-b_{1}, \\
b_{2}=\frac{133}{10}+N_{a}, & b_{3} \equiv \frac{10}{3}-5 x+\frac{5 x^{2}}{12}+\left(\left(N^{2}-1\right) \xi_{1}^{\prime 2}+N \xi_{2}^{\prime 2}\right) \frac{3 x^{2}}{2}, & b_{1}=\frac{196}{45}+N_{c} .
\end{array}
$$

\section{$8 \mathrm{SU}(9)$ fixed points}

The $\mathrm{SU}(N)$ discussion proceeds in the same manner as the $\mathrm{SO}(N)$ case. In ref. [26] we showed that the minimum value of $N$ consistent with the existence of a UVFP is $N=9$, when $b_{g}^{\min }$ is $4 / 3$. For the reduced couplings $x_{i}=\lambda_{i} / \alpha$ we found a flat space UVFP with

$$
\begin{aligned}
& x_{1}=0.386000, \quad x_{2}=0.293121, \quad x_{3}=0.502429, \\
& x_{4}=0.195158, \quad x_{5}=0.398832 .
\end{aligned}
$$

Just as in the $\mathrm{SO}(N)$ case, when gravitational corrections are included, we find a UVFP with similar $x_{i}$ values to the above, a large value of $x$, and tiny nonzero values for $\xi_{1}^{\prime}$ and $\xi_{2}^{\prime}$. 


\begin{tabular}{|c|c|c|c|c|c|}
\hline$\widetilde{b}_{g}$ & $\widetilde{y}_{1}$ & $\widetilde{y}_{2}$ & $\widetilde{y}_{3}$ & $\widetilde{y}_{4}$ & $\widetilde{y}_{5}$ \\
\hline 0. & 2.64270 & 0.275255 & 0.289413 & 0.970346 & 0.371374 \\
\hline $1 / 3$ & 2.94605 & 0.284989 & 0.312552 & 1.20422 & 0.389234 \\
\hline $1 / 2$ & 3.15683 & 0.290153 & 0.325788 & 1.39047 & 0.398894 \\
\hline $3 / 4$ & 3.67495 & 0.298306 & 0.348280 & 1.94791 & 0.414424 \\
\hline 0.84798 & 4.36728 & 0.301646 & 0.358128 & 2.99190 & 0.420885 \\
\hline
\end{tabular}

Table 3. UVFPs for $\mathrm{SU}(\infty)$.

\section{$9 \mathrm{SU}(N)$ large $N$ limit}

We can define a large $N$ limit in a similar way to the $\mathrm{SO}(N)$ case. Once again we set

$$
\alpha=\frac{\widetilde{\alpha}}{N}, \lambda_{1}=\frac{\widetilde{\lambda}_{1}}{N^{2}}, \lambda_{2}=\frac{\tilde{\lambda}_{2}}{N}, \lambda_{3}=\frac{\widetilde{\lambda}_{3}}{N}, \lambda_{4}=\frac{\widetilde{\lambda}_{4}}{N^{2}}, \lambda_{5}=\frac{\widetilde{\lambda}_{5}}{N} .
$$

As before there are distinct options for the rescaling of the gravitational corrections.

\subsection{Gravitational coupling rescaling-A}

With identical rescalings to those described in the corresponding $\mathrm{SO}(N)$ case, we find for the reduced quartic couplings $\widetilde{y}_{i} \equiv \widetilde{\lambda}_{i} / \tilde{g}^{2}$,:

$$
\begin{aligned}
& \bar{\beta}_{\widetilde{y}_{1}}=\widetilde{y}_{1}^{2}+12 \widetilde{y}_{2}^{2}+18-\left(12-\widetilde{b}_{g}-8 \widetilde{y}_{2}\right) \widetilde{y}_{1}, \\
& \bar{\beta}_{\widetilde{y}_{2}}=4 \widetilde{y}_{2}^{2}+3-\left(12-\widetilde{b}_{g}\right) \widetilde{y}_{2}, \\
& \bar{\beta}_{\widetilde{y}_{3}}=2 \widetilde{y}_{3}^{2}+\frac{1}{2} \widetilde{y}_{5}^{2}+\frac{3}{2}-\left(6-\widetilde{b}_{g}\right) \widetilde{y}_{3}, \\
& \bar{\beta}_{\widetilde{y}_{4}}=\widetilde{y}_{5}\left(\widetilde{y}_{1}+2 \widetilde{y}_{2}+2 \widetilde{y}_{3}\right)+\widetilde{y}_{5}^{2}+3+\widetilde{y}_{4}\left(\widetilde{y}_{1}+4 \widetilde{y}_{2}+2 \widetilde{y}_{3}-\left(9-\widetilde{b}_{g}\right)\right), \\
& \bar{\beta}_{\widetilde{y}_{5}}=\widetilde{y}_{5}^{2}+3-\left(9-\widetilde{b}_{g}-2 \widetilde{y}_{2}\right) \widetilde{y}_{5} .
\end{aligned}
$$

Just as in the $\mathrm{SO}(N)$ case, any flat space UVFP would necessarily imply a corresponding FP with $\widetilde{\xi_{1}^{\prime}}=\widetilde{\xi}_{2}^{\prime}=x=0$. We found such FPs for specific values of $\widetilde{b}_{g}$ in ref. [26], as shown in table 3 .

Interestingly, eq. (9.2) becomes identical to eq. (5.3), the corresponding set of results in the $\mathrm{SO}(N)$ case, if we do the following:

- Replace $\widetilde{y}_{5}$ by $\widetilde{y}_{5} / 2$

- Replace $\widetilde{b}_{g}$ by $2 \widetilde{b}_{g}$

- Multiply the r.h.s. of all of eq. (9.2a)-eq. (9.2e) by 2 . 


\begin{tabular}{|c|c|c|c|c|c|c|c|}
\hline$\widetilde{b}_{g}$ & $\widetilde{y}_{1}$ & $\widetilde{y}_{2}$ & $\widetilde{y}_{3}$ & $\widetilde{y}_{4}$ & $\widetilde{y}_{5}$ & $x$ & $\widetilde{\xi}_{2}^{\prime}$ \\
\hline 0.8192 & 4.79737 & 0.30066 & 0.35517 & 3.90016 & 0.41896 & $\widetilde{b}_{2} /\left(\widetilde{\xi}_{1}^{\prime}\right)^{2}$ & $1.88636 \widetilde{\xi}_{1}$ \\
\hline 0.40961 & 4.79737 & 0.30066 & 2.23523 & -3.19276 & 0.41896 & $\widetilde{b}_{2} /\left(\widetilde{\xi}_{1}^{\prime}\right)^{2}$ & $1.88636 \widetilde{\xi}^{\prime}{ }_{1}$ \\
\hline
\end{tabular}

Table 4. Curved space UVFPs for $\mathrm{SU}(\infty)$.

At first sight, this result is surprising but can be understood (or at least made plausible), by inspection of the respective Dynkin diagrams. This means, of course that the FPs are essentially identical in the two cases. This is immediately apparent in the comparison of the first two rows of table 1 with the corresponding rows in table 3 . (Recall that $\widetilde{b}_{g}=1 / 6$ in the $\mathrm{SO}(N)$ case corresponds to $\widetilde{b}_{g}=1 / 3$ in the $\mathrm{SU}(N)$ case).

In the same limit the gravitational couplings are:

$$
\begin{aligned}
& \bar{\beta}_{\widetilde{a}}=\widetilde{a}\left(\widetilde{b}_{g}-\widetilde{b}_{2} \widetilde{a}\right), \\
& \bar{\beta}_{\widetilde{x}}=-\widetilde{a} \widetilde{x}\left(\frac{3}{2} \widetilde{\xi}_{1}^{2} \widetilde{x}-\widetilde{b}_{2}\right), \\
& \bar{\beta}_{\widetilde{\xi}_{1}^{\prime}}=\left(\widetilde{y}_{1}+4 \widetilde{y}_{2}-6\right) \widetilde{\xi}_{1}^{\prime}, \\
& \bar{\beta}_{\widetilde{\xi}_{2}^{\prime}}=(2 \widetilde{y}-33) \widetilde{\xi}_{2}^{\prime}+\left(\widetilde{y}_{4}+\frac{1}{2} \widetilde{y}_{5}\right) \widetilde{\xi}_{1}^{\prime} .
\end{aligned}
$$

As we remarked above, any flat space UVFP would necessarily imply a corresponding FP with $\widetilde{\xi}_{1}^{\prime}=\widetilde{\xi_{2}^{\prime}}=x=0$, but one destabilised by eq. (9.3b). However, once again we see from eq. (9.3b) that such a FP is unstable with respect to fluctuations in $x$.

Now let us seek a FP for nonzero $\widetilde{\xi}_{1}^{\prime}$. Just as in the $\mathrm{SO}(N)$ case, we find two real FPs, as shown in table 4 .

These results are essentially identical to the corresponding ones for $\mathrm{SO}(N)$; because, of course, the large $N \beta$-functions in the two cases are essentially identical, differing only by redefinitions of the coupling $\widetilde{y}_{5}$ and overall rescaling. Once again we do not find any real FPs in this case.

\subsection{Gravitational coupling rescaling-B}

We again have the possibility of a nontrivial alternative rescaling, as described for $\mathrm{SO}(\mathrm{N})$ in section 5.2. With eq. (5.5) we find (in the large $N$ limit):

$$
\begin{aligned}
& \bar{\beta}_{\widetilde{y}_{1}}=\widetilde{y}_{1}^{2}+12 \widetilde{y}_{2}^{2}+18-\left(12-\widetilde{b}_{g}-8 \widetilde{y}_{2}\right) \widetilde{y}_{1}+5 \widetilde{a}^{2}{\widetilde{\xi_{1}^{\prime}}}^{2}, \\
& \bar{\beta}_{\widetilde{y}_{2}}=4 \widetilde{y}_{2}^{2}+3-\left(12-\widetilde{b}_{g}\right) \widetilde{y}_{2}, \\
& \bar{\beta}_{\widetilde{y}_{3}}=2 \widetilde{y}_{3}^{2}+\frac{1}{2} \widetilde{y}_{5}^{2}+\frac{3}{2}-\left(6-\widetilde{b}_{g}\right) \widetilde{y}_{3}, \\
& \bar{\beta}_{\widetilde{y}_{4}}=\widetilde{y}_{5}\left(\widetilde{y}_{1}+2 \widetilde{y}_{2}+2 \widetilde{y}_{3}\right)+\widetilde{y}_{5}^{2}+3+\widetilde{y}_{4}\left(\widetilde{y}_{1}+4 \widetilde{y}_{2}+2 \widetilde{y}_{3}-\left(9-\widetilde{b}_{g}\right)\right)+5 \widetilde{a}^{2} \widetilde{\xi}_{1}^{\prime} \widetilde{\xi}_{2}^{\prime}, \\
& \bar{\beta}_{\widetilde{y}_{5}}=\widetilde{y}_{5}^{2}+3-\left(9-\widetilde{b}_{g}-2 \widetilde{y}_{2}\right) \widetilde{y}_{5},
\end{aligned}
$$




$$
\begin{aligned}
& \bar{\beta}_{\widetilde{\xi}_{1}^{\prime}}=\left(\widetilde{y}_{1}+4 \widetilde{y}_{2}-6\right) \widetilde{\xi}_{1}^{\prime}+\frac{10}{3} \frac{\widetilde{a} \widetilde{\xi}_{1}^{\prime}}{\widetilde{x}} \\
& \bar{\beta}_{\widetilde{\xi}_{2}^{\prime}}=\frac{1}{36}\left(\left(-54+36 \widetilde{y}_{3}\right) \widetilde{\xi}_{2}^{\prime}+18 \widetilde{\xi}_{1}^{\prime}\left(\widetilde{y}_{4}+\frac{1}{2} \widetilde{y}_{5}\right)\right)+\frac{10}{3} \frac{\widetilde{a} \widetilde{\xi}_{2}^{\prime}}{\widetilde{x}} \\
& \bar{\beta}_{\widetilde{x}}=-(1 / 12) \widetilde{a}\left(18 \widetilde{x}^{2}{\widetilde{\xi_{1}^{\prime}}}^{2}-12 \widetilde{b}_{2} \widetilde{x}+40\right) .
\end{aligned}
$$

Here, although the $\beta$-functions in eq. (9.4a)-eq. (9.4f) can be converted to the corresponding $\mathrm{SO}(N)$ results by redefining $y_{5}, \widetilde{a} \xi_{1,2}^{\prime}$, and $x$, we cannot then redefine eq. (9.4g) in the same way. Nevertheless, we see that, in this case also, the natural generalisation of the UVFP we identified in flat space (corresponding to $\widetilde{\xi}_{1}^{\prime}=\widetilde{\xi}_{2}^{\prime}=0$ ) is destabilised by the gravitational corrections.

\section{Dimensional transmutation in the $\mathrm{SO}(N)$ model}

In either the $\mathrm{SO}(N)$ or the $\mathrm{SU}(N)$ case, a comprehensive analysis of the behaviour of the effective action with two distinct scalar multiplets and five independent quartic scalar couplings would be a formidable undertaking. We choose to make the crucial assumption that DT occurs via the development of a vev for the adjoint representation only, just as we analysed in ref. [25]. The precise details differ, however, because the analysis involves the behaviour of the couplings under renormalisation, and the renormalisation of the adjoint self couplings are, of course, affected by the presence of the other multiplet.

In ref. [25] we focused our attention on the $\mathrm{SO}(10)$ theory. We showed it was asymptotically free both without and with gravitational interactions, and that in the latter case, the adjoint developed a vev via DT, with symmetry breaking uniquely determined to be $\mathrm{SO}(10) \rightarrow \mathrm{SU}(5) \otimes \mathrm{U}(1)$. With the addition of a multiplet of scalars in the fundamental representation, we showed in ref. [26] that asymptotic freedom is not sustained in the flat space case; the minimum value of $N$ necessary becomes $N=12$. We have seen above that this remains true when gravitational interactions are included. Therefore we need to generalise our previous discussion to accommodate $N>10$.

We begin by assuming that the background metric is well-approximated by the de Sitter metric,

$$
R_{\alpha \beta \gamma \delta}=\frac{R}{12}\left(g_{\alpha \gamma} g_{\beta \delta}-g_{\alpha \delta} g_{\beta \gamma}\right),
$$

with constant $R>0$. Then if $\Phi$ is constant and non-zero, we showed in ref. [25] that, in the $\mathrm{SO}(10)$ case, symmetry breaking occurs in the $\mathrm{SU}(5) \otimes \mathrm{U}(1)$ direction with

$$
\frac{\Phi}{\sqrt{N R}}=r\left(\begin{array}{rr}
1 & 0 \\
0 & -1
\end{array}\right),
$$

where 1 is the $5 \otimes 5$ identity matrix, and $r \equiv \sqrt{2 T_{2} /(N R)}=\sqrt{\left(\phi^{a} \phi^{a}\right) /(N R)}$.

It is easy to see that if we assume that $\chi$ does not get a vev, then for any even $N, \Phi$ takes the same block form, that is proportional to

$$
r\left(\begin{array}{rr}
1 & 0 \\
0 & -1
\end{array}\right)
$$


with $N / 2 \times N / 2$ blocks. The residual symmetry after such spontaneous symmetry breaking $(\mathrm{SSB})$ is $\mathrm{SU}(N / 2) \otimes \mathrm{U}(1)$ for arbitrary $r$. Thus the classical action for (even $N$ ) takes the off-shell value

$$
\frac{S_{\mathrm{cl}}\left(\lambda_{i}, r\right)}{V_{4}}=\frac{1}{3 b}+\frac{c}{6}+\frac{\zeta_{1}}{2}\left(N r^{2}\right)^{2}-N r^{2} \xi_{1},
$$

where $V_{4}$ is an angular volume, and $\left\{\lambda_{i}\right\}$ is the complete set of couplings. Here, we introduce the symbol $\zeta_{1}$ for the sum of the first two couplings,

$$
\zeta_{1} \equiv \lambda_{1}+2 \lambda_{2} / N
$$

In order for the action per unit volume to be bounded from below, we must have $\zeta_{1}>0$. We shall also need the derivatives

$$
\frac{S_{\mathrm{cl}}^{\prime}}{V_{4}}=2 N r\left[N \zeta_{1} r^{2}-\xi_{1}\right], \quad \frac{S_{\mathrm{cl}}^{\prime \prime}}{V_{4}}=2 N\left[3 N \zeta_{1} r^{2}-\xi_{1}\right] .
$$

Here (and subsequently)

$$
S_{\mathrm{cl}}^{\prime} \equiv \frac{\partial S_{\mathrm{cl}}\left(\lambda_{i}, r\right)}{\partial r}
$$

We can determine the extremal values of $r$ at tree level by solving $S_{\mathrm{cl}}^{\prime}=0$. Therefore, $r=0$ or $r=r_{0}$ with

$$
r_{0}^{2} \equiv \frac{\xi_{1}}{N \zeta_{1}}
$$

which, since $\zeta_{1}>0$, yields $r_{0}$ real only if $\xi_{1}>0$. Assuming this requirement is satisfied, the classical curvature $S_{\mathrm{cl}}^{\prime \prime}$ is negative at $r=0$, so the unbroken solution is a maximum. At $r_{0}$, the curvature takes the value

$$
\frac{S_{\mathrm{cl}}^{\prime \prime}(o s)}{V_{4}}=4 N \xi_{1}>0 .
$$

At the minimum, the value of the classical action is

$$
\frac{S_{\mathrm{cl}}^{(\mathrm{os})}}{V_{4}}=\frac{1}{3 b}+\frac{c}{6}-\frac{\xi_{1}^{2}}{2 \zeta_{1}} .
$$

Recall that, for $N=12$, the UVFP, eq. (4.3), has $\xi_{1}^{\prime} \approx 0$, or $\xi_{1} \approx-1 / 6$. This is a generic result, so it is important to establish that it is possible to fulfill this condition and still have $\xi_{1}>0$ at the DT scale. (This did in fact occur the simpler model [25] that did not include the scalar $\chi$ in the fundamental.)

Generally, we want to determine whether DT can occur. Including radiative corrections, the effective action takes the generic form

$$
\Gamma\left(\lambda_{i}, r, \rho / \mu\right)=S_{\mathrm{cl}}\left(\lambda_{i}, r\right)+B\left(\lambda_{i}, r\right) \log (\rho / \mu)+\frac{C\left(\lambda_{i}, r\right)}{2} \log ^{2}(\rho / \mu)+\ldots,
$$

where $\rho \equiv \sqrt{R}$. All coupling constants are denoted by the set $\left\{\lambda_{i}\right\}$. In writing the effective action in this form, we have assumed that $\Phi$ is spacetime independent; it is not necessary to assume that the breaking pattern is to $\mathrm{SU}(N / 2) \otimes \mathrm{U}(1)$. 
We seek an extremum of the effective action such that $(r, \rho)=(\langle r\rangle,\langle\rho\rangle) \equiv\left(r_{0}, v\right)$, i.e., solutions to

$$
\begin{aligned}
\left.\frac{\partial}{\partial r} \Gamma\left(\lambda_{i}, r, \rho / \mu\right)\right|_{r_{0}, v} & =\left.\frac{\partial}{\partial r} S_{\mathrm{cl}}\left(\lambda_{i}, r\right)\right|_{r_{0}, v}=0, \\
\left.\rho \frac{\partial}{\partial \rho} \Gamma\left(\lambda_{i}, r, \rho / \mu\right)\right|_{r_{0}, v} & =\left.B\left(\lambda_{i}, r\right)\right|_{r_{0}, v}=0,
\end{aligned}
$$

where we choose $\mu=\langle\rho\rangle \equiv v$. These results are exact to all orders in the loop expansion.

In order to determine stability, we shall also need the matrix of second derivatives on-shell:

$$
\begin{aligned}
\left.\frac{\partial^{2}}{\partial r^{2}} \Gamma\left(\lambda_{i}, r, \rho / \mu\right)\right|_{r_{0}, v} & =\left.\frac{\partial^{2}}{\partial r^{2}} S_{\mathrm{cl}}\left(\lambda_{i}, r\right)\right|_{r_{0}, v}, \\
\left.\rho \frac{\partial^{2}}{\partial r \partial \rho} \Gamma\left(\lambda_{i}, r, \rho / \mu\right)\right|_{r_{0}, v} & =\left.\frac{\partial}{\partial r} B\left(\lambda_{i}, r\right)\right|_{r_{0}}, \\
\left.\rho^{2} \frac{\partial^{2}}{\partial \rho^{2}} \Gamma\left(\lambda_{i}, r, \rho / \mu\right)\right|_{r_{0}, v} & =C\left(\lambda_{i}, r_{0}\right) .
\end{aligned}
$$

As before, we implicitly set $\mu=v$ after performing the derivatives. The second variation on-shell can be written

$$
\delta^{(2)} \Gamma=\frac{1}{2}\left(\begin{array}{ll}
\frac{\delta \rho}{\rho} \delta r
\end{array}\right)\left[\begin{array}{ll}
C\left(\lambda_{i}, r_{0}\right) & B^{\prime}\left(\lambda_{i}, r_{0}\right) \\
B^{\prime}\left(\lambda_{i}, r_{0}\right) & S_{m}^{\prime \prime}\left(\lambda_{i}, r_{0}\right)
\end{array}\right]\left(\begin{array}{c}
\frac{\delta \rho}{\rho} \\
\delta r
\end{array}\right) .
$$

Given our conventions, these equations eq. (10.13) are also exact to all orders in the loop expansion, but their leading nonzero contributions vary from tree level for those involving $S_{\mathrm{cl}}$, to one-loop for $B$, to two-loop ${ }^{7}$ for $C$. This is the characteristic "see-saw" pattern, so this matrix has two eigenvalues $\varpi_{i}$ that may be approximated as

$$
\varpi_{1}\left(r_{0}, v\right)=\frac{S_{\mathrm{cl}}^{\prime \prime}}{2}+O\left(\hbar^{2}\right), \quad \varpi_{2}\left(r_{0}, v\right)=\frac{1}{2}\left[C_{2}-\frac{\left(B_{1}^{\prime}\right)^{2}}{S_{\mathrm{cl}}^{\prime \prime}}\right]+O\left(\hbar^{3}\right) .
$$

$\varpi_{1}$ is determined by the classical curvature and given by eq. (10.9), and $\varpi_{2}$, although of order $\hbar^{2}$, is determined by one-loop results.

In ref. [21], we used the renormalisation group to show that (to leading order)

$$
B_{1}=\sum_{i} \beta_{\lambda_{i}} \frac{\partial S_{\mathrm{cl}}}{\partial \lambda_{i}}
$$

so that the conditions for an extremum corresponding to DT in this model (and others of this general form) are $r=r_{0}$ and

$$
\left.B_{1}\right|_{r=r_{0}}=B_{1}^{(\mathrm{os})}=\left.\sum_{i} \beta_{\lambda_{i}} \frac{\partial S_{\mathrm{cl}}}{\partial \lambda_{i}}\right|_{r=r_{0}}=\sum_{i} \beta_{\lambda_{i}} \frac{\partial S_{\mathrm{cl}}^{(\mathrm{os})}}{\partial \lambda_{i}}=0
$$

\footnotetext{
${ }^{7}$ However, the two-loop contribution to $C_{2}$ can be calculated from one-loop corrections; to $C_{3}$, from two-loop corrections, etc.
} 
where $B_{1}^{(\text {os })}, S_{\mathrm{cl}}^{(\mathrm{os})}$ are the "on-shell" contributios to $B_{1}, S_{\mathrm{cl}}$ with "on-shell" corresponding to $r=r_{0}$. Notice that

$$
\frac{\partial S_{\mathrm{cl}}^{(\mathrm{os})}}{\partial \lambda_{i}}=\left.\left[\frac{\partial S_{\mathrm{cl}}}{\partial \lambda_{i}}+\frac{\partial S_{\mathrm{cl}}}{\partial r} \frac{\partial r}{\partial \lambda_{i}}\right]\right|_{r=r_{0}}=\left.\frac{\partial S_{\mathrm{cl}}}{\partial \lambda_{i}}\right|_{r=r_{0}} .
$$

Such an extremum corresponds to a minimum if $\zeta_{1}>0$ and $\varpi_{2}>0$, and we showed, again using the renormalisation group, that [21, 24, 25]

$$
\varpi_{2}=\left.\frac{1}{2}\left[\beta_{\lambda_{i}}^{(1)} \frac{\partial B_{1}}{\partial \lambda_{i}}-\frac{1}{S_{\mathrm{cl}}^{\prime \prime}}\left(\beta_{\lambda_{i}}^{(1)} \frac{\partial}{\partial \lambda_{i}} S_{\mathrm{cl}}^{\prime}\left(\lambda_{i}, r\right)\right)^{2}\right]\right|_{r_{0}, v} .
$$

or using eq. (10.16),

$$
\varpi_{2}=\left.\frac{1}{2}\left[\left(\beta_{\lambda_{i}}^{(1)} \frac{\partial}{\partial \lambda_{i}}\right)^{2}\left[S_{\mathrm{cl}}\right]-\frac{1}{S_{\mathrm{cl}}^{\prime \prime}}\left(\beta_{\lambda_{i}}^{(1)} \frac{\partial}{\partial \lambda_{i}} S_{\mathrm{cl}}^{\prime}\left(\lambda_{i}, r\right)\right)^{2}\right]\right|_{r_{0}, v}
$$

Remarkably, $\varpi_{2}$ can also be written

$$
\varpi_{2}=\frac{1}{2}\left[\beta_{\lambda_{i}}^{(1)} \frac{\partial B_{1}^{o s}}{\partial \lambda_{i}}\right]
$$

an observation which is not particularly obvious and that we shall explain in detail elsewhere in a more general discussion of the RG and effective actions in this kind of theory.

From eq. (10.17), we find

$$
B_{1}\left(\lambda_{i}, r\right)=\frac{b_{3}\left(x, \xi_{1}^{\prime}, \xi_{2}^{\prime}\right)}{3 x^{2}}-\frac{b_{1}}{6}+\frac{1}{2}\left(\alpha N r^{2}\right)^{2}\left(\bar{\beta}_{z_{1}}-b_{g} z_{1}\right)-\left(\alpha N r^{2}\right) \bar{\beta}_{\xi_{1}^{\prime}}
$$

where $b_{1}, b_{3}$ are given in eq. (3.7b), and $z_{1}=\zeta_{1} / \alpha$, where $\zeta_{1}$ was defined in eq. (10.5). (Obviously, $\bar{\beta}_{\xi_{1}^{\prime}}=\bar{\beta}_{\xi_{1}}$.)

$$
B_{1}^{(\mathrm{os})}=\frac{b_{3}\left(x, \xi_{1}^{\prime}, \xi_{2}^{\prime}\right)}{3 x^{2}}-\frac{b_{1}}{6}+\left(\frac{\xi_{1}}{z_{1}}\right)\left[\frac{1}{2}\left(\frac{\xi_{1}}{z_{1}}\right)\left(\bar{\beta}_{z_{1}}-b_{g} z_{1}\right)-\bar{\beta}_{\xi_{1}^{\prime}}\right]
$$

This rather succinct formula hides a good deal of complexity in the expressions for $\bar{\beta}_{z_{1}}=$ $\bar{\beta}_{x_{1}}+2 \bar{\beta}_{x_{2}} / N$ (see eq. (A.1c) for the $N=12$ case), and for $\bar{\beta}_{\xi_{1}^{\prime}}$, eqs. (3.14), (3.15). We shall leave further discussion of the determination of the extrema from $B_{1}^{(\mathrm{os})}=0$ and the flow from such an extremum towards the UVFP to the next section, section 11.

\section{Dimensional transmutation in the $\mathrm{SO}(12)$ model}

Even after restriction to $N=12, b_{g}=1 / 6$, this relatively simple model is still extremely complicated, both analytically and numerically, for several reasons. In our classically scaleinvariant version, it involves many fields, and only the possible "directions" of SSB in this field space is determined, depending on relations among the 10 dimensionless coupling constants of the bosonic sector. The scale of SSB is determined at one-loop order, and 
the determination of its character (and correspondingly, the dilaton mass) is determined at two-loops. The numerical results for the UV behavior were given in section 4, showing that such a model can be AF for a certain range of coupling constants.

We now wish to show that the model can undergo DT and that it is locally stable for at least a portion of the DT surface. This will require fleshing out eq. (10.23) in greater detail. From eq. (3.7b), we have for $N=12$,

$$
b_{3}=\frac{10}{3}-5 x+\frac{5 x^{2}}{12}+9\left(11 \xi_{1}^{\prime 2}+2 \xi_{2}^{2}\right) x^{2},
$$

As mentioned in section 4 , even specifying $b_{g}=1 / 6$ does not yield a unique model, since several different arrangements of the fermion content are still possible. In section 4, we arbitrarily chose to focus on the case of 52 two-component fermions in the fundamental representation $N$ so that $N_{f}=312$ and $b_{1}=917 / 36, b_{2}=59$. As noted earlier, as the running scale decreases, we must hope that $\xi_{1}^{\prime}$ will run from near zero to the region where $\xi_{1}=\xi_{1}^{\prime}-1 / 6>0$. For this reason, exploring the DT surface may be simpler in terms of $\xi_{1}$ rather than $\xi_{1}^{\prime}$. Further, $B_{1}$ depends on the couplings $\left(x_{4}, x_{5}\right)$ only via the linear combination $^{8} x_{4}+x_{5} / 24 \equiv z_{4}$. After making these notational changes, we find that $B_{1}^{(\mathrm{os})}$, eq. (10.23), becomes

$$
\begin{aligned}
B_{1}^{(\mathrm{os})}= & \frac{10}{9 x^{2}}-\frac{5}{3 x}-\frac{689}{216}+\frac{\xi_{1}}{3}\left(6 \xi_{1}-1\right)+6 \xi_{2}^{\prime 2}+\frac{\xi_{1}}{z_{1}}\left[5-\frac{35 x_{2}}{18}-12 \xi_{2}^{\prime} z_{4}\right] \\
& +\frac{\xi_{1}^{2}}{z_{1}^{2}}\left[5+\frac{35 x_{2}^{2}}{18}+6 z_{4}^{2}\right]+\frac{\bar{a} \xi_{1}^{2}}{2 z_{1}}\left[5+\frac{x}{6}-\frac{20}{3 x}-x \xi_{1}\left(12 \xi_{1}+1\right)\right] \\
& +\frac{\bar{a}^{2} \xi_{1}^{4}}{2 z_{1}^{2}}\left[5+\frac{x^{2}}{4}+3 x^{2} \xi_{1}\left(3 \xi_{1}+1\right)\right] .
\end{aligned}
$$

We note that $z_{1}$ enters only as the ratio $\xi_{1} / z_{1}$, and $\bar{a}$ appears only as the combination $\bar{a} \xi_{1}^{2} / z_{1}$, which may well turn out to be very small.

To determine $\varpi_{2}$, we need to evaluate either eqs. (10.19), (10.20) or eq. (10.21) for $N=12$. From eq. (11.2), we see that, when expressed in terms of the 7 rescaled variables $\left\{\bar{a}, x, \xi_{1}, \xi_{2}^{\prime}, z_{1}, x_{2}, z_{4}\right\}, B_{1}^{(\mathrm{os})}$ is independent of $\left\{x_{3}, x_{5}\right\}$. Consequently, we require 7 reduced $\beta$-functions. The actual determination of $\varpi_{2}$ is unavoidably complicated and not very illuminating. For completeness, In appendix A, we give the relevant $\beta$-functions and the resulting exact formula for $\varpi_{2}$.

For our purpose here, we shall be satisfied to demonstrate a region of parameter space such that $\varpi_{2}>0$ and our other requirements (such as $\xi_{1}>0$ ) are satisfied and that the couplings there proceed to run to the UVFP. (A complete description of solutions of $B_{1}^{\text {(os) }}=0$ remains a formidable undertaking). The simplest way to identify such a region is to start with as many couplings as possible (consistent with requirements at the DT scale) already near the UVFP. The most obvious problem with this is that, since we plan to generate the E-H term using the adjoint vev, we require $\xi_{1}>0$, whereas at the UVFP $\xi_{1} \approx-1 / 6$. We can however choose to try $\xi_{2}^{\prime}$ at or near zero.

In table 5 , we give two examples of points satisfying $B_{1}=0, \varpi_{2}>0$, and flowing to the UVFP.

\footnotetext{
${ }^{8}$ For general $\left.N, z_{4} \equiv x_{4}+x_{5} /(2 N)\right)$.
} 


\begin{tabular}{|c|c|c|c|c|c|c|c|}
\hline$\widetilde{x}_{1}$ & $\widetilde{x}_{2}$ & $\widetilde{x}_{3}$ & $\widetilde{x}_{4}$ & $\widetilde{x}_{5}$ & $x$ & $\widetilde{\xi}_{1}$ & $\widetilde{\xi}_{2}^{\prime}$ \\
\hline 0.373073 & 0.25 & 0.377518 & 0.25 & 0.582159 & 148.271 & 0.190359 & 0.042969 \\
\hline 0.3577213 & 0.25 & 0.377518 & 0.25 & 0.582159 & 120.471 & 0.184093 & 0 \\
\hline
\end{tabular}

Table 5. DT points in the UVFP catchment basin.

\section{Summary and conclusions}

This paper continues a series in which we have developed the theory of renormalisable quantum gravity (RQG) coupled to matter fields, including in particular a clear demonstration that generation of scalar vacuum expectation values can occur via Dimensional Transmutation (DT). We have also claimed to identify a large class of such theories that exhibit asymptotic freedom, and consequently represent a possible UV completion of Einstein gravity (in combination with DT). This claim remains controversial because of disagreement [20] concerning the correct sign of the coefficient $b$ of the $R^{2}$ term in the Lagrangian, eq. (2.2). We believe that the sign we adopt (which results in AF for the $b$-coupling) is required for convergence of the Path Integral.

In another previous paper [26], we addressed the issue of $\mathrm{AF}$ in $\mathrm{SU}(N)$ and $\mathrm{SO}(N)$ gauge theories in flat space in the presence of adjoint and fundamental scalar representations. We identified some errors in the original treatment, and also added a discussion of a large $N$ limit, where for a limited range of (small) values of $\tilde{b}_{g}$ we showed that a UVFP existed.

In this paper we generalise this work by coupling these theories to RQG. In both the $\mathrm{SO}(N)$ and $\mathrm{SU}(N)$ cases, the inclusion of these interactions has essentially no effect on the minimum value of $N$ required for a UVFP, nor in the basic features of this FP. We described the $\mathrm{SO}(10)$ case (with only an adjoint scalar) in detail in ref. [25]. With both scalar representations, however, the minimum value of $N$ for AF becomes $N=12 .{ }^{9}$ We argued, that in the $\mathrm{SO}(12)$ case, the theory with both scalar representations would exhibit a region of parameter space consistent with Dimensional Transmutation, in a similar way to the case with an adjoint only, and, moreover, that from parts of this region, the couplings flowed to a UVFP (which we identified) at high energies. In appendix A, we present the expression for $\varpi_{2}$, defined in eqs. (10.19)-(10.21), which is required to be positive within a subregion of the DT surface.

The large $N$ limit is interesting. We identified two distinct ways to implement the limit for the gravitational couplings. In both cases, however, the UVFP that we identified in flat space is destabilized by inclusion of these couplings.

To sum up: we have demonstrated that it is feasible to construct a realistic Grand Unified Theory with a non-trivial set of scalar field representations (adjoint+scalar) for both $\mathrm{SU}(N)$ and $\mathrm{SO}(N)$ cases, with complete asymptotic freedom and (we argue) Dimensional Transmutation to a low energy theory with gravitational self-interactions described

\footnotetext{
${ }^{9}$ In the case of $\mathrm{SU}(N)$, the minimum value of $N$ for a UVFP is $N=7$ for the theory with only the adjoint scalar, becoming $N=9$ in the presence of both adjoint and fundamental.
} 
by the Einstein term. However the cases $\mathrm{SO}(10)$ and $\mathrm{SU}(5)$ are excluded. Moreover, the minimum value of $N$ required is higher for both $\mathrm{SU}(N)$ and $\mathrm{SO}(N)$ than in the case with only an adjoint scalar, making it not unlikely that it will be higher still with a more complicated set of scalar representations. Problems with the scenario remain, most obviously the generation of the electroweak scale, and the issue of unitarity, which we hope to address elsewhere [28].

\section{Acknowledgments}

DRTJ thanks KITP (Santa Barbara), where part of this work was done, for hospitality, and the Leverhulme Trust for the award of an Emeritus Fellowship. This research was supported in part by the National Science Foundation under Grant No. NSF PHY11-25915, by the Leverhulme Trust, and by the University of Liverpool.

\section{A Reduced $\beta$-functions and result for $\varpi_{2}$}

With reference to section 11 , the relevant $\beta$-functions for $N=12, b_{g}=1 / 6$ are

$$
\begin{aligned}
\bar{\beta}_{\bar{a}}= & 59 \bar{a}\left(\frac{1}{354}-\bar{a}\right) \\
\bar{\beta}_{x}= & \bar{a}\left(-\frac{10}{3}+64 x-x^{2}\left(\frac{5}{12}+9\left(11\left(\xi_{1}+\frac{1}{6}\right)^{2}+2 \xi_{2}^{\prime 2}\right)\right)\right) \\
\bar{\beta}_{z_{1}}= & \bar{a}^{2} \xi_{1}^{2}\left(5+9 x^{2}\left(\xi_{1}+\frac{1}{6}\right)^{2}\right)+\bar{a} z_{1}\left(5-18 x\left(\xi_{1}+\frac{1}{6}\right)^{2}\right)+74 z_{1}^{2} \\
& +\frac{z_{1}}{6}\left(140 x_{2}-359\right)+\frac{35 x_{2}^{2}}{9}+12 z_{4}^{2}+10 \\
\bar{\beta}_{x_{2}}= & \bar{a} x_{2}\left(5-18 x\left(\xi_{1}+\frac{1}{6}\right)^{2}\right)+21 x_{2}^{2}+x_{2}\left(12 z_{1}-\frac{359}{6}\right)+\frac{x_{5}^{2}}{8}+6 \\
\bar{\beta}_{z_{4}}= & \bar{a}^{2} \xi_{1}\left(\xi_{2}^{\prime}-\frac{1}{6}\right)\left(5+9 x^{2}\left(\xi_{1}+\frac{1}{6}\right) \xi_{2}^{\prime}\right)+\bar{a} z_{4}\left(5-3 x\left(\left(\xi_{1}+\frac{1}{6}\right)^{2}+4\left(\xi_{1}+\frac{1}{6}\right) \xi_{2}^{\prime}+\xi_{2}^{\prime 2}\right)\right) \\
& +4 z_{4}^{2}+z_{4}\left(68 z_{1}+14 x_{3}+\frac{35 x_{2}}{3}-\frac{139}{3}\right)+\frac{35 x_{5}^{2}}{144}+\frac{5}{2} \\
\bar{\beta}_{\xi_{1}}= & \bar{a} \xi_{1}\left(\frac{10}{3 x}-x\left(\xi_{1}+\frac{1}{6}\right)\left(3 \xi_{1}+2\right)\right)+\left(\xi_{1}+\frac{1}{6}\right)\left(68 z_{1}+\frac{35 x_{2}}{3}-30\right)+12 \xi_{2}^{\prime} z_{4} \\
\bar{\beta}_{\xi_{2}^{\prime}=} & \bar{a}\left(\xi_{2}^{\prime}-\frac{1}{6}\right)\left(\frac{10}{3 x}-\frac{3}{2} x \xi_{2}^{\prime}\left(1+2 \xi_{2}^{\prime}\right)\right)+\xi_{2}^{\prime}\left(14 x_{3}-\frac{33}{2}\right)+12\left(\xi_{1}+\frac{1}{6}\right) z_{4} .
\end{aligned}
$$

Since $x_{3}$ and $x_{5}$ do not appear in $B_{1}^{(\text {os })}$, eq. (11.2), we have omitted $\bar{\beta}_{x_{3}}$ and $\bar{\beta}_{x_{5}}$ above. Here, as with $B_{1}^{(\text {os) }}$, we have employed an asymmetric notation in terms of $\left\{\xi_{1}, \xi_{2}^{\prime}\right\}$. 
$\varpi_{2}$ is $\alpha$ times a function of all 9 rescaled couplings. (Recall our convention is $\alpha \equiv g^{2}$.) After restoring factors of $16 \pi^{2}$, the result of the evaluation of eq. (10.21) is ${ }^{10}$

$$
\begin{aligned}
& \varpi_{2}=\frac{\alpha}{512 \pi^{4}}\left[\frac{5}{3}\left(7 x_{2}-18\right)\left(4 \xi_{1}^{2}-\frac{35}{18}\right)+6 \xi_{2}^{2}\left(28 x_{3}-33\right)-\bar{a}^{4} \frac{\xi_{1}^{6}}{z_{1}^{3}}\left(5+\frac{x^{2}\left(6 \xi_{1}+1\right)^{2}}{4}\right)^{2}\right. \\
& +\bar{a}^{3} \frac{\xi_{1}^{4}}{z_{1}^{2}}\left(\frac{50}{x}-\frac{5}{8} x^{2}\left(6 \xi_{1}+1\right)^{2}+\frac{5}{12} x\left(6 \xi_{1}+1\right)\left(72 \xi_{1}+1\right)-\frac{665}{2}\right. \\
& \left.-\frac{1}{48} x^{3} \xi_{1}^{4}\left(6 \xi_{1}+1\right)^{2}\left(41+402 \xi_{1}+1008 \xi_{1}^{2}+216 \xi_{2}^{\prime 2}\right)\right) \\
& +\frac{34}{9}\left(6 \xi_{1}+1\right)\left(12 \xi_{1}-1\right) z_{1}-8 \xi_{2}^{\prime} z_{4}+\xi_{1}\left(96 \xi_{2}^{\prime} z_{4}-40-\frac{70 x_{2}}{9}\right) \\
& -\frac{\xi_{1}^{2}}{81 z_{1}^{3}}\left(108 z_{4}^{2}+35 x_{2}^{2}+90\right)^{2} \\
& +\frac{\xi_{1}}{648 z_{1}^{2}}\left(8\left(35 x_{2}+216 \xi_{2}^{\prime} z_{4}-90\right)\left(108 z_{4}^{2}+35 x_{2}^{2}+90\right)\right. \\
& +3 \xi_{1}\left(7840 x_{2}^{3}-25200 x_{2}^{2}+105 x_{2}\left(x_{5}^{2}-192\right)\right. \\
& \left.\left.+18\left(5 z_{4}\left(7 x_{5}^{2}+72\right)+72\left(28 x_{3}-33\right) z_{4}^{2}+576 z_{4}^{3}+3580\right)\right)\right) \\
& +\bar{a}^{2}\left(\frac { \xi _ { 1 } ^ { 2 } } { z _ { 1 } } \left(\frac{1330}{3 x}-\frac{100}{3 x^{2}}+\frac{5 x}{12}\left(6 \xi_{1}+1\right)\left(12 \xi_{1}-1\right)\right.\right. \\
& -\frac{5}{18}\left(611+12 \xi_{1}+432 \xi_{1}^{2}-36 \xi_{2}^{\prime}+432 \xi_{2}^{\prime 2}\right) \\
& \left.+\frac{x^{2}}{18}\left(6 \xi_{1}+1\right)\left(1719 \xi_{1}^{2}+5184 \xi_{1}^{3}+27 \xi_{2}^{\prime 2}-324 \xi_{2}^{\prime 3}+9 \xi_{1}\left(13+36 \xi_{2}^{\prime 2}\right)-5\right)\right) \\
& -\frac{\xi_{1}^{4}}{z_{1}^{3}}\left(\frac{10}{9}\left(108 z_{4}^{2}+35 x_{2}^{2}+90\right)+\frac{x^{2}}{18}\left(6 \xi_{1}+1\right)^{2}\left(108 z_{4}^{2}+35 x_{2}^{2}+90\right)\right) \\
& +\frac{\xi_{1}^{3}}{z_{1}^{2}}\left(\frac{5}{6}\left(35 x_{2}+12 z_{4}\left(24 \xi_{2}^{\prime}-1\right)-90\right)\right. \\
& +\frac{x^{2}}{24}\left(6 \xi_{1}+1\right)\left(35 x_{2}+60 \xi_{1}^{2}\left(7 x_{2}-18\right)+8 \xi_{1}\left(35 x_{2}+216 \xi_{2}^{\prime} z_{4}-90\right)\right. \\
& \left.\left.\left.+18\left(8 \xi_{2}^{\prime} z_{4}\left(3 \xi_{2}^{\prime}+1\right)-5\right)\right)\right)\right) \\
& +\frac{1}{z_{1}}\left(\xi_{1}^{2}\left(\frac{70}{3} x_{2}^{2}-48 z_{4}^{2}-60\right)-\frac{1}{324}\left(35 x_{2}+216 \xi_{2}^{\prime} z_{4}-90\right)^{2}+\xi_{1}\left(\frac{1505}{6}+\frac{700 x_{2}^{2}}{27}\right.\right. \\
& \left.\left.-\frac{35 x_{5}^{2}}{144}+4 z_{4}^{2}-\frac{1}{12} \xi_{2}^{\prime}\left(35 x_{5}^{2}+72\left(5-66 z_{4}+56 x_{3} z_{4}+8 z_{4}^{2}\right)\right)\right)\right) \\
& +\bar{a}\left(\frac{200}{27 x^{3}}-\frac{1330}{9 x^{2}}-\frac{5}{18}\left(19-6 \xi_{1}+36 \xi_{1}^{2}+108 \xi_{2}^{\prime 2}\right)\right. \\
& +\frac{10}{27 x}\left(307-9 \xi_{1}+72 \xi_{1}^{2}-18 \xi_{2}^{\prime}+216 \xi_{2}^{2}\right)
\end{aligned}
$$

\footnotetext{
${ }^{10}$ For readers who would prefer this formula in Mathematica or Maple format, we have provided these online as supplementary material.
} 


$$
\begin{aligned}
& +x\left(2 \xi_{1}-\frac{37 \xi_{1}^{2}}{3}-346 \xi_{1}^{3}-1200 \xi_{1}^{4}-3 \xi_{2}^{\prime 2}\left(6 \xi_{2}^{\prime}-1\right)\left(2 \xi_{2}^{\prime}+1\right)\right) \\
& +\frac{1}{z_{1}}\left(\frac{5}{9 x} \xi_{1}\left(90-35 x_{2}+12\left(1-24 \xi_{2}^{\prime}\right) z_{4}\right)+\frac{5}{18} \xi_{1}\left(35 x_{2}+36\left(6 \xi_{2}^{\prime} z_{4}-5\right)\right)\right. \\
& \quad+x\left(-20 \xi_{1}^{4}\left(7 x_{2}-18\right)-\frac{2}{3} \xi_{1}^{3}\left(70 x_{2}+648 \xi_{2}^{\prime} z_{4}-315\right)\right. \\
& \left.\quad+\xi_{1}^{2}\left(25+\frac{35}{18} x_{2}+48 \xi_{2}^{\prime} z_{4}\left(3 \xi_{2}^{\prime}-1\right)\right)+\xi_{1}\left(\frac{35}{36} x_{2}+2 \xi_{2}^{\prime} z_{4}\left(18 \xi_{2}^{\prime}+36 \xi_{2}^{\prime 2}-1\right)\right)\right) \\
& +\frac{\xi_{1}^{2}}{z_{1}^{2}}\left(\frac{20\left(108 z_{4}^{2}+35 x_{2}^{2}+90\right)}{27 x}-\frac{5}{18}\left(35 x_{2}^{2}+54\left(2 z_{4}^{2}+5\right)\right)\right. \\
& \quad+\frac{x}{108}\left(90-175 x_{2}^{2}-1296 z_{4}^{2} \xi_{2}^{\prime}\left(3 \xi_{2}^{\prime}+2\right)+36 \xi_{1}^{2}\left(630+35 x_{2}^{2}+648 z_{4}^{2}\right)\right. \\
& \left.\left.\left.\left.\quad-24 \xi_{1}\left(35 x_{2}^{2}+18\left(9\left(4 \xi_{2}^{\prime}-1\right) z_{4}^{2}-10\right)\right)\right)\right)\right)\right] .
\end{aligned}
$$

Open Access. This article is distributed under the terms of the Creative Commons Attribution License (CC-BY 4.0), which permits any use, distribution and reproduction in any medium, provided the original author(s) and source are credited.

\section{References}

[1] K.S. Stelle, Renormalization of higher derivative quantum gravity, Phys. Rev. D 16 (1977) 953 [INSPIRE].

[2] E.S. Fradkin and A.A. Tseytlin, Renormalizable asymptotically free quantum theory of gravity, Phys. Lett. 104B (1981) 377 [INSPIRE].

[3] E.S. Fradkin and A.A. Tseytlin, Renormalizable asymptotically free quantum theory of gravity, Nucl. Phys. B 201 (1982) 469 [InSPIRE].

[4] I.G. Avramidi and A.O. Barvinsky, Asymptotic freedom in higher derivative quantum gravity, Phys. Lett. 159B (1985) 269 [inSPIRE].

[5] I.L. Buchbinder, S.D. Odintsov and I.L. Shapiro, Effective action in quantum gravity, IOP, Bristol U.K. (1992).

[6] I.G. Avramidi, Heat kernel and quantum gravity, Lect. Notes Phys. Monogr. 64 (2000) 1 [INSPIRE].

[7] T.P. Cheng, E. Eichten and L.-F. Li, Higgs phenomena in asymptotically free gauge theories, Phys. Rev. D 9 (1974) 2259 [InSPIRE].

[8] I.L. Buchbinder et al., The stability of asymptotic freedom in grand unified models coupled to $R^{2}$ gravity, Phys. Lett. B 216 (1989) 127 [InSPIRE].

[9] I.L. Buchbinder et al., Asymptotically free grand unification models with quantum $R^{2}$ gravitation, Sov. J. Nucl. Phys. 49 (1989) 544 [Yad. Fiz. 49 (1989) 876] [inSPIRE].

[10] S. Weinberg, Ultraviolet divergences in quantum theories of gravitation, in General relativity: an Einstein centenary survey, S.W. Hawking and W. Israel, Cambridge University Press, Cambridge U.K. (1979). 
[11] M.R. Niedermaier, Gravitational fixed points from perturbation theory, Phys. Rev. Lett. 103 (2009) 101303 [InSPIRE].

[12] M. Niedermaier, Gravitational fixed points and asymptotic safety from perturbation theory, Nucl. Phys. B 833 (2010) 226 [inSPIRE].

[13] A. Eichhorn, Status of the asymptotic safety paradigm for quantum gravity and matter, Found. Phys. 48 (2018) 1407 [arXiv: 1709.03696] [INSPIRE].

[14] N. Christiansen, D.F. Litim, J.M. Pawlowski and M. Reichert, Asymptotic safety of gravity with matter, Phys. Rev. D 97 (2018) 106012 [arXiv:1710.04669] [INSPIRE].

[15] K.G. Falls, D.F. Litim and J. Schröder, Aspects of asymptotic safety for quantum gravity, Phys. Rev. D 99 (2019) 126015 [arXiv:1810.08550] [INSPIRE].

[16] R. Percacci, An introduction to covariant quantum gravity and asymptotic safety, World Scientific, Singapore (2017).

[17] E.S. Fradkin and A.A. Tseytlin, Asymptotic freedom in extended conformal supergravities, Phys. Lett. B 110 (1982) 117.

[18] E.S. Fradkin and A.A. Tseytlin, Conformal anomaly in Weyl theory and anomaly free superconformal theories, Phys. Lett. B 134 (1984) 187.

[19] E.S. Fradkin and A.A. Tseytlin, Conformal supergravity, Phys. Rept. 119 (1985) 233 [INSPIRE].

[20] A. Salvio and A. Strumia, Agravity, JHEP 06 (2014) 080 [arXiv:1403.4226] [INSPIRE].

[21] M.B. Einhorn and D.R.T. Jones, Naturalness and dimensional transmutation in classically scale-invariant gravity, JHEP 03 (2015) 047 [arXiv: 1410.8513] [INSPIRE].

[22] S.R. Coleman and E.J. Weinberg, Radiative corrections as the origin of spontaneous symmetry breaking, Phys. Rev. D 7 (1973) 1888 [INSPIRE].

[23] G.F. Giudice, G. Isidori, A. Salvio and A. Strumia, Softened gravity and the extension of the Standard Model up to infinite energy, JHEP 02 (2015) 137 [arXiv:1412.2769] [INSPIRE].

[24] M.B. Einhorn and D.R.T. Jones, Induced gravity I: real scalar field, JHEP 01 (2016) 019 [arXiv: 1511.01481] [INSPIRE].

[25] M.B. Einhorn and D.R.T. Jones, Induced gravity II: grand unification, JHEP 05 (2016) 185 [arXiv: 1602.06290] [INSPIRE].

[26] M.B. Einhorn and D.R.T. Jones, Asymptotic freedom in certain $\mathrm{SO}(N)$ and $\mathrm{SU}(N)$ models, Phys. Rev. D 96 (2017) 055035 [arXiv:1705.00751] [INSPIRE].

[27] M.B. Einhorn and D.R.T. Jones, Renormalizable, asymptotically free gravity without ghosts or tachyons, Phys. Rev. D 96 (2017) 124025 [arXiv:1710.03795] [InSPIRE].

[28] M.B. Einhorn and D.R.T. Jones, A first-order formalism for renormalizable quantum gravity, in preparation. 